This item was submitted to Loughborough's Research Repository by the author.

Items in Figshare are protected by copyright, with all rights reserved, unless otherwise indicated.

\title{
Numerical continuation analysis of a dual-sidestay main landing gear mechanism
}

\section{PLEASE CITE THE PUBLISHED VERSION}

http://dx.doi.org/10.2514/1.C032130

\section{PUBLISHER}

(c) American Institute of Aeronautics and Astronautics

\section{VERSION}

AM (Accepted Manuscript)

\section{PUBLISHER STATEMENT}

This work is made available according to the conditions of the Creative Commons Attribution-NonCommercialNoDerivatives 4.0 International (CC BY-NC-ND 4.0) licence. Full details of this licence are available at: https://creativecommons.org/licenses/by-nc-nd/4.0/

\section{LICENCE}

CC BY-NC-ND 4.0

\section{REPOSITORY RECORD}

Knowles, James A.C., Bernd Krauskopf, Mark H. Lowenberg, Simon A. Neild, and P. Thota. 2015. "Numerical Continuation Analysis of a Dual-sidestay Main Landing Gear Mechanism". figshare.

https://hdl.handle.net/2134/18692. 


\title{
Numerical Continuation Analysis of a Dual-sidestay Main Landing Gear Mechanism
}

\author{
James A. C. Knowles* \\ Oxford Brookes University, Oxford, OX33 1HX, UK \\ Bernd Krauskopf ${ }^{\dagger}$ \\ Mark H. Lowenberg $\ddagger$ and Simon A. Neild ${ }^{\ddagger}$ \\ The University of Auckland, Auckland 1142, NZ \\ University of Bristol, Bristol, BS8 1TR, UK \\ P. Thota ${ }^{\S}$ \\ Airbus in the UK, Bristol, BS99 7AR, UK
}

\begin{abstract}
A model of a three-dimensional dual-sidestay landing gear mechanism is presented and employed in an investigation of the sensitivity of the downlocking mechanism to attachment point deflections. A motivation for this study is the desire to understand the underlying nonlinear behaviour, which may prevent a dual-sidestay landing gear from downlocking under certain conditions. The model formulates the mechanism as a set of steady-state constraint equations. Solutions to these equations are then continued numerically in state and parameter space, providing all state parameter dependencies within the model from a single computation. The capability of this analysis approach is demonstrated with an investigation into the effects of the aft sidestay angle on retraction actuator loads. It was found that the retraction loads are not significantly affected by the sidestay plane angle, but the landing gear's ability to be retracted fully is impeded at certain sidestay plane angles. This result is attributed to the landing gear's geometry, as the locklinks are placed under tension and cause the mechanism to lock. Sidestay flexibilities and attachment point deflections are then introduced to enable the downlock loads to be investigated. The investigation into the dual sidestay's downlock sensitivity to attachment point deflections yields an underlying double hysteresis loop, which is highly sensitive to these deflections. Attachment point deflections of a few millimetres were found to prevent the locklinks from automatically downlocking under their own weight, hence requiring some external force to downlock the landing gear. Sidestay stiffness was also found to influence the downlock loads, although not to the extent of attachment point deflection.
\end{abstract}

${ }^{*}$ Lecturer in Mechanical Engineering, Department of Mechanical Engineering and Mathematical Sciences, Wheatley Campus, Wheatley, Oxford, OX33 1HX, UK

${ }^{\dagger}$ Professor of Applied Mathematics, Department of Mathematics, Private bag 92019, Auckland 1142, NZ

${ }^{\ddagger}$ Readers in Dynamics, Faculty of Engineering, Queen’s Building, University Walk, Bristol, BS8 1TR, UK

$\S$ Model Developer - Physical Systems, Airbus in the UK, New Filton House, Filton, Bristol, BS99 7AR, UK 


\section{Introduction}

Conventional Main Landing Gears (MLGs) have a single-sidestay to support the shock strut when the gear experiences side loads (e.g. under high-speed cornering on the ground). Some MLGs may have a second structural drag stay to support the shock strut against aerodynamic drag loading. Others feature an angled sidestay to absorb both types of loads (lateral ground and aerodynamic drag loads). With the increasing use of new materials (such as carbon fibre composites) in new aircraft primary structural elements, landing gear designs are having to evolve to meet new design constraints. Whilst composite materials offer large potential weight savings due to their high strength, they are not as good as metals at absorbing point loads. This provides a challenge when integrating the landing gear into a carbon fibre wing-box section, because the attachment points (where the landing gear meets the wing-box) transfer very large loads into the airframe. In order to be able to integrate the landing gear into a carbon fibre wing, the loads at the attachment points must be reduced. One solution could be to increase the number of landing gears on the aircraft, thus reducing the load on each gear when the aircraft is manoeuvring on the ground. An alternative solution, which has been adopted by both Boeing ${ }^{1}$ and Airbus $^{2}$ for the main landing gears on both of their latest aircraft, is to add a second sidestay into the mechanism; this is referred to as a dual-sidestay main landing gear (DSS MLG). The presence of two sidestays spreads the loads transfered from the gear to the wingbox, allowing the DSS MLG to be integrated into a carbon fibre wing.

Whilst DSS MLGs provide a solution to integrating a landing gear into a composite wing structure, the nature of the DSS mechanism presents challenges in itself due to its sensitivity to changes in MLG parameters, such as attachment point positions and aerodynamic drag. The mechanism is particularly sensitive to these parameters around the downlock point, which is the state of the landing gear defined as separating the 'unlocked' and 'downlocked' states. In this state the two locklink links align with one another, and at the same time the upper and lower sidestay links are also very close to aligning. The reasons for the sensitivity of DSS MLGs near the downlock point are not fully understood.

The literature on landing gear mechanism analysis is limited and relatively old, ${ }^{3,4}$ and it focuses on the kinematic aspects of the landing gear mechanism from a preliminary design perspective. There are currently no examples in the public domain of DSS MLG mechanism modelling. The vast majority of previous work into landing gear modelling has tended to focus on capturing the landing gear properties under ground loading ${ }^{5-8}$ by building relatively complex dynamic models using dynamic simulation software packages (such as Dymola or ADAMS). These models are very good at capturing many different aspects of the physical system, and they can provide quantitatively accurate results for a specific system of interest. On the other hand, complex dynamic models are less suitable to developing an understanding of general underlying nonlinear behaviour. This is because the model parameters that can be simulated continuously 
within the model are often limited to externally applied forces. If, for example, the MLG geometry was to be investigated, the model would need to be adjusted and multiple time histories conducted for different (discrete) geometries. Not only is this a time-consuming process, but areas of highly nonlinear behaviour may be missed if a relevant geometry is not simulated.

The approach presented here expresses the mechanism as a set of steady-state constraint equations, which are solved simultaneously with the method of numerical continuation. Tools from Bifurcation Theory, ${ }^{9-11}$ including numerical continuation, have been used to help understand nonlinear problems in Aerospace applications before. ${ }^{12-14}$ For all of these applications, numerical continuation was shown to provide significant advantages over alternative analysis methods. This paper outlines a mechanism modelling approach that enables the use of numerical continuation methods to analyse DSS MLG mechanisms. The following section briefly describes the model; it is self-contained and builds on our previous work modelling single-stay NLG and MLG mechanisms. ${ }^{12,15}$ A formulation validation is then presented by starting from the case of a singlesidestay MLG and then 'rotating out' an extra sidestay. Subsequently, continuation results for a DSS MLG are presented with an emphasis on downlock sensitivity to sidestay flexibility. The final section presents some concluding remarks and offers an outlook on future model advancements that could be introduced to increase the applicability of the results.

\section{Model details}

The model used in this work was derived using newtonian mechanical principles. Because of this, the equations within this section are presented in their entirety.

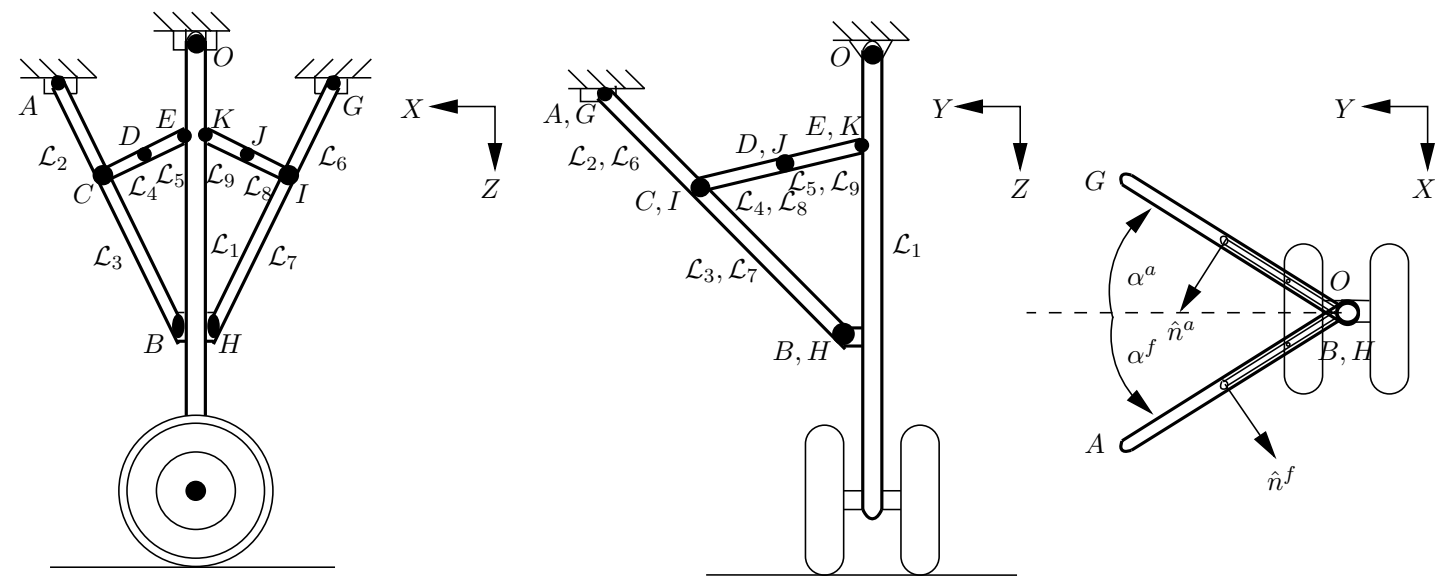

Figure 1: Three-view of a symmetrical DSS MLG arrangement, with joints, locklinks and sidestay plane normal vectors shown.

Figure 1 shows the DSS MLG geometry considered, with a main vertical shock strut supported by two 
folding sidestays. For the landing gear considered in this work, the rake angle is taken to be zero. The sidestays are attached to the shock strut at points slightly offset from the shock strut centreline. The other end of each sidestay is attached to the airframe at points $A$ and $G$. The locklinks, attached between the sidestay joints and the shock strut, lock the gear in position when deployed. Locklink configurations differ from landing gear to landing gear; one of the locklinks on the Boeing 787 DSS MLG, for example, is attached to the sidestays and the airframe (rather than the sidestays and the shock strut as considered here). The model of the DSS MLG considered here is a development of the single sidestay MLG model formulation presented previously; ${ }^{15}$ as such, the notation follows a similar convention. As Figure 1 shows, the DSS MLG mechanism consists of nine links, which are initially assumed to be rigid bodies with uniformly distributed mass along their lengths. Each link, $\mathcal{L}_{i}$, is connected to another link or the aircraft structure via rotational joints; the majority of the joints labelled in Figure 1 are planar joints, with the exception of joints $A, B$, $G$ and $H$ which are spherical joints that allow connected bodies to rotate about the joint freely in threedimensions. The $X$-axis is defined as the shock strut rotation axis, with the shock strut rotation joint at the global co-ordinate origin point $O$. The gear is defined to retract in the positive $(Y, Z)$-plane and the $Z$-axis is aligned with the global gravity vector, positive down.

Due to the presence of two sidestays, two transformation matrices are required to define the two sidestay rotation planes - a fore and aft plane. These two transformation matrices are defined in terms of two normal vectors $\hat{n}^{f}$ and $\hat{n}^{a}$ for the fore and aft sidestay planes, respectively, as given by:

$$
\begin{aligned}
& \hat{n}^{f}=\overline{O A} \times \overline{O B} \\
& \hat{n}^{a}=\overline{O G} \times \overline{O H}
\end{aligned}
$$

The two sidestay local co-ordinate systems can now be defined with two rotation matrices. The fore rotation matrix $T^{f}$ describes rotations about the global origin point $\mathrm{O}$, which aligns the local fore $x$-axis $\left(x^{f}\right)$ with $\hat{n}^{f}$ by a rotation over $\alpha^{f}$ about the global $Y$-axis, followed by a rotation through $\beta^{f}$ about the intermediate $z$-axis:

$$
T^{f}=\left[\begin{array}{ccc}
\cos \beta^{f} \cos \alpha^{f} & -\sin \beta^{f} & \cos \beta^{f} \sin \alpha^{f} \\
\sin \beta^{f} \cos \alpha^{f} & \cos \beta^{f} & \sin \beta^{f} \sin \alpha^{f} \\
-\sin \alpha^{f} & 0 & \cos \alpha^{f}
\end{array}\right]
$$

The aft rotation matrix $T^{a}$ is also a transformation about the global origin point O, but one that aligns the local aft $x$-axis $\left(x^{a}\right)$ with $\hat{n}^{a}$ by a rotation over $\alpha^{a}$ about the global $Y$-axis followed by a rotation through $\beta^{a}$ about the intermediate $z$-axis: 


$$
T^{a}=\left[\begin{array}{ccc}
\cos \beta^{a} \cos \alpha^{a} & -\sin \beta^{a} & \cos \beta^{a} \sin \alpha^{a} \\
\sin \beta^{a} \cos \alpha^{a} & \cos \beta^{a} & \sin \beta^{a} \sin \alpha^{a} \\
-\sin \alpha^{a} & 0 & \cos \alpha^{a}
\end{array}\right] .
$$

The two local co-ordinate systems are therefore related to the global $(X, Y, Z)$ co-ordinates as follows:

$$
\begin{aligned}
& {\left[\begin{array}{c}
x^{f} \\
y^{f} \\
z^{f}
\end{array}\right]=T^{f}\left[\begin{array}{c}
X \\
Y \\
Z
\end{array}\right],} \\
& {\left[\begin{array}{c}
x^{a} \\
y^{a} \\
z^{a}
\end{array}\right]=T^{a}\left[\begin{array}{c}
X \\
Y \\
Z
\end{array}\right] .}
\end{aligned}
$$

The equations are formulated by considering each link $\mathcal{L}_{i}$ within the mechanism as an individual rigid body in static equilibrium. This method has been previously introduced, ${ }^{12,15}$ and is now extended to the case of a DSS MLG.

\section{A. Link description and co-ordinate systems}

Figure 2 depicts the general naming convention used for each link within the landing gear mechanism in local fore (a) and aft (b) co-ordinates. Each link is described in terms of seven elements, $\mathcal{L}_{i}=\left\{X_{i}, Y_{i}, Z_{i}, \hat{n}, \theta_{i}, L_{i}, m_{i}\right\}$, where:

- $\mathcal{L}_{i}$ is the $i^{\text {th }}$ link;

- $X_{i}, Y_{i}, Z_{i}$ are the global Cartesian co-ordinates which describe the position of $\mathcal{L}_{i}$ 's centre of gravity $(\mathrm{cg})$;

- $\hat{n}$ is the normal vector to $\mathcal{L}_{i}$ 's plane of rotation, i.e. perpendicular to the page in Figure 2;

- $\theta_{i}$ is the local rotation of $\mathcal{L}_{i}$ relative to the local y-axis ${ }^{\mathrm{a}}$;

- $L_{i}$ is the length of $\mathcal{L}_{i}$;

- $m_{i}$ is the mass of $\mathcal{L}_{i}$, assumed to be evenly distributed along $\mathcal{L}_{i}$.

${ }^{a}$ For the main strut $\mathcal{L}_{1}$ a global rotation $\Theta_{1}$ is used to define the link: see Figure 3 for graphical representation. The corresponding local rotations $\theta_{1}^{f}$ and $\theta_{1}^{a}$ (shown in Figure 2) are functions of $\Theta_{1}$ 


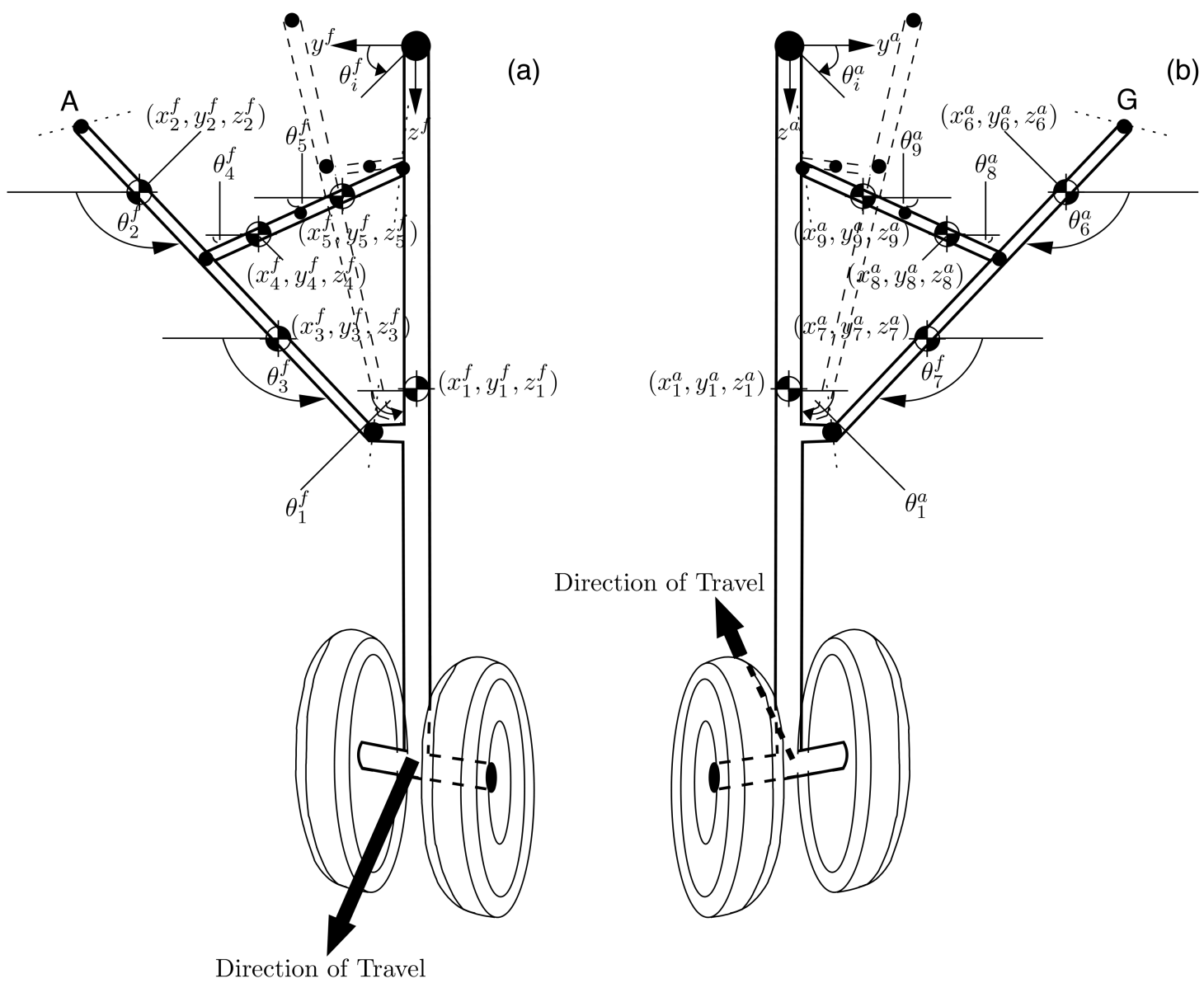

Figure 2: Naming convention shown in two local $(y, z)$ co-ordinate systems, looking along the normal vectors to the fore (a) and aft (b) planes. The direction of travel for the aircraft is shown in both cases.

The forces acting on $\mathcal{L}_{i}$ can be expressed in global, and in local fore or aft co-ordinates. The fore and aft co-ordinate systems are related to the global co-ordinates by the fore and aft transformation matrices, respectively, as:

$$
\begin{aligned}
& {\left[\begin{array}{c}
F^{x f} \\
F^{y f} \\
F^{z f}
\end{array}\right]=T^{f}\left[\begin{array}{l}
\mathcal{F}^{X} \\
\mathcal{F}^{Y} \\
\mathcal{F}^{Z}
\end{array}\right],} \\
& {\left[\begin{array}{c}
F^{x a} \\
F^{y a} \\
F^{z a}
\end{array}\right]=T^{a}\left[\begin{array}{l}
\mathcal{F}^{X} \\
\mathcal{F}^{Y} \\
\mathcal{F}^{Z}
\end{array}\right] .}
\end{aligned}
$$

The left-hand sides of Equations (5a) and (5b) are the local projections of the given force, with the symbol $F$ used to distinguish the force as being in local co-ordinates; the right-hand side of the equations 
contains the global $(X, Y, Z)$ projections of the same force, again denoted by the symbol $\mathcal{F}$ to distinguish it as being a global force projection.

\section{B. Geometric constraints}

A system of 35 geometric constraint equations is needed to express the physical constraints in the DSS MLG mechanism: of these, three equations are expressed in global co-ordinates $\left(X_{i}, Y_{i}, Z_{i}\right)$, along with a single rotation $\Theta_{i}$ about the global $X$-axis; 16 equations are formulated using the local fore sidestay co-ordinate

cg positions $\left(x_{i}^{f}, y_{i}^{f}, z_{i}^{f}\right)$, along with a single rotation $\theta_{i}^{f}$ in the local fore plane; 16 equations are formulated using the local aft sidestay co-ordinate cg positions $\left(x_{i}^{a}, y_{i}^{a}, z_{i}^{a}\right)$, along with a single rotation $\theta_{i}^{a}$ in the local aft plane. The 35 geometric constraint equations obtained are:

$$
\left.\begin{array}{c}
{\left[\begin{array}{c}
X_{1} \\
Y_{1}-\frac{L_{1}}{2} \cos \left(\Theta_{1}\right) \\
Z_{1}-\frac{L_{1}}{2} \sin \left(\Theta_{1}\right)
\end{array}\right]=0,} \\
x_{2}^{f} \\
y_{2}^{f}-\frac{L_{2}}{2} \cos \left(\theta_{2}^{f}\right)-A^{y^{f}} \\
z_{2}^{f}-\frac{L_{2}}{2} \sin \left(\theta_{2}^{f}\right)-A^{z^{f}} \\
x_{3}^{f} \\
y_{2}^{f}-y_{3}^{f}+\frac{L_{2}}{2} \cos \left(\theta_{2}^{f}\right)+\frac{L_{3}}{2} \cos \left(\theta_{3}^{f}\right) \\
z_{2}^{f}-z_{3}^{f}+\frac{L_{2}}{2} \sin \left(\theta_{2}^{f}\right)+\frac{L_{3}}{2} \sin \left(\theta_{3}^{f}\right) \\
y_{3}^{f}-y_{1}^{f}+\frac{L_{3}}{2} \cos \left(\theta_{3}^{f}\right)+l_{13} \cos \left(\theta_{1}^{f}+\omega_{1}^{f}\right) \\
z_{3}^{f}-z_{1}^{f}+\frac{L_{3}}{2} \sin \left(\theta_{3}^{f}\right)+l_{13} \sin \left(\theta_{1}^{f}+\omega_{1}^{f}\right) \\
x_{4}^{f} \\
y_{5}^{f}-y_{1}^{f}+\frac{L_{5}}{2} \cos \left(\theta_{5}^{f}\right)-l_{15} \cos \left(\theta_{1}^{f}+\omega_{2}^{f}\right) \\
y_{4}^{f}-z_{1}^{f}+\frac{L_{5}}{2} \sin \left(\theta_{5}^{f}\right)-l_{15} \sin \left(\theta_{1}^{f}+\omega_{2}^{f}\right)
\end{array}\right]=0,
$$




$$
\left[\begin{array}{c}
x_{6}^{a} \\
y_{6}^{a}-\frac{L_{6}}{2} \cos \left(\theta_{6}^{a}\right)-G^{y^{a}} \\
z_{6}^{a}-\frac{L_{6}}{2} \sin \left(\theta_{6}^{a}\right)-G^{z^{a}} \\
x_{7}^{a} \\
y_{6}^{a}-y_{7}^{a}+\frac{L_{6}}{2} \cos \left(\theta_{6}^{a}\right)+\frac{L_{7}}{2} \cos \left(\theta_{7}^{a}\right) \\
z_{6}^{a}-z_{7}^{a}+\frac{L_{6}}{2} \sin \left(\theta_{6}^{a}\right)+\frac{L_{7}}{2} \sin \left(\theta_{7}^{a}\right) \\
y_{7}^{a}-y_{1}^{a}+\frac{L_{7}}{2} \cos \left(\theta_{7}^{a}\right)+l_{17} \cos \left(\theta_{1}^{a}+\omega_{3}^{a}\right) \\
z_{7}^{a}-z_{1}^{a}+\frac{L_{7}}{2} \sin \left(\theta_{7}^{a}\right)+l_{17} \sin \left(\theta_{1}^{a}+\omega_{3}^{a}\right) \\
x_{8}^{a} \\
y_{9}^{a}-y_{1}^{a}+\frac{L_{9}}{2} \cos \left(\theta_{9}^{a}\right)-l_{19} \cos \left(\theta_{1}^{a}+\omega_{4}^{a}\right) \\
z_{9}^{a}-z_{1}^{a}+\frac{L_{9}}{2} \sin \left(\theta_{9}^{a}\right)-l_{19} \sin \left(\theta_{1}^{a}+\omega_{4}^{a}\right) \\
z_{8}^{a}-\frac{L_{6}}{2} \cos \left(\theta_{6}^{a}\right)+\frac{L_{8}}{2} \cos \left(\theta_{8}\right) \\
y_{9}^{a}-\frac{L_{6}}{2} \sin \left(\theta_{6}^{a}\right)+\frac{L_{8}}{2} \sin \left(\theta_{8}\right) \\
x_{9}^{a}+\frac{L_{9}}{2} \cos \left(\theta_{9}^{a}\right)+\frac{L_{8}}{2} \operatorname{sos}\left(\theta_{8}^{a}\right)
\end{array}\right]
$$

Here $A^{y^{f}}, A^{z^{f}}, G^{y^{a}}$ and $G^{z^{a}}$ are the local co-ordinate $y$ - and $z$-components of the sidestay attachment points (points $A$ and $G$ in Figure 1), $l_{13}, l_{15}, l_{17}$ and $l_{19}$ are the lengths from the shock strut cg to the adjoining ends of links $\mathcal{L}_{3}, \mathcal{L}_{5}, \mathcal{L}_{7}$ and $\mathcal{L}_{9}$, respectively, and $\omega_{1}^{f}$ and $\omega_{2}^{f}, \omega_{3}^{a}$ and $\omega_{4}^{a}$ are the angles $l_{13}, l_{15}, l_{17}$ and $l_{19}$ make with the shock strut centreline (in the appropriate local co-ordinates). All other symbols follow the naming convention that capital letters indicate global co-ordinates and lower cases indicate local co-ordinates, with superscripts ' $f$ ' and ' $a$ ' distinguishing between fore and aft local co-ordinate systems.

\section{Force and Moment Equilibrium Equations}

The 35 geometric constraints are supplemented with a second set of 37 equations that describe the force and moment equilibrium necessary for the gear to be in a steady-state. For the whole DSS MLG to be in equilibrium, each of the nine links must be in force and moment equilibrium and the joints must also be in force equilibrium. The links are acted upon by internal forces, which in global co-ordinates are denoted by $\mathcal{F}_{i ; *}$ in the general case. The subscript $i$ denotes the link number that the force is acting on and the subscript $*$ denotes the element exerting that force on $\mathcal{L}_{i}$ which can be either another link, $\mathcal{L}_{*}$, or the aircraft body. If the aircraft body is responsible for exerting the force, then the subscript symbol $*$ is replaced by 
a subscript $R A, R O$ or $R G$, depending on if the reaction force acts at points $A, O$ or $G$ on the landing gear (respectively). Unlike the single sidestay MLG model, ${ }^{15}$ the DSS MLG requires more than one local co-ordinate system, so it is necessary to distinguish between force projections in the local fore and local aft planes. This distinction is made in a similar manner to that of the local positional co-ordinates, by using superscripts ' $f$ ' and ' $a$ ' such that:

$$
\begin{aligned}
& F_{i ; *}^{f}=T^{f} \mathcal{F}_{i ; *}, \\
& F_{i ; *}^{a}=T^{a} \mathcal{F}_{i ; *} .
\end{aligned}
$$

Within the sidestay plane, for an arbitrary link $\mathcal{L}_{i}$ to be in static equilibrium the sum of the forces acting on the link must equal zero, along with the sum of the moments about an arbitrary point $P$. Using the notation conventions as before, this means that:

$$
\begin{aligned}
& \sum_{*} F_{i ; *}^{y f}=0, \quad \sum_{*} F_{i ; *}^{z f}=0, \quad \sum_{*} M_{i ; *}^{P f}=l_{i ; *}^{P f} F_{i ; *}^{f}=0, \\
& \sum_{*} F_{i ; *}^{y a}=0, \quad \sum_{*} F_{i ; *}^{z a}=0, \quad \sum_{*} M_{i ; *}^{P a}=l_{i ; *}^{P a} F_{i ; *}^{a}=0 .
\end{aligned}
$$

From Equations (8a), the equilibrium equations for links $\mathcal{L}_{2}-\mathcal{L}_{5}$ can be formulated as follows: for link $\mathcal{L}_{2}$, the moment equilibrium equation is

$$
-F_{2 ; 3,4}^{y} L_{2} \sin \theta_{2}^{f}+F_{2 ; 3,4}^{z} L_{2} \cos \theta_{2}^{f}=\frac{L_{2}}{2} m_{2} g_{z} \cos \theta_{2}^{f}-\frac{L_{2}}{2} m_{2} g_{y} \sin \theta_{2}^{f}
$$

for link $\mathcal{L}_{3}$, the force equilibrium equations are

$$
\begin{aligned}
& F_{3 ; 2,4}^{y}+F_{3 ; 1}^{y}=m_{3} g_{y}, \\
& F_{3 ; 2,4}^{z}+F_{3 ; 1}^{z}=m_{3} g_{z},
\end{aligned}
$$

and the moment equilibrium equation is

$$
-F_{3 ; 1}^{y} L_{3} \sin \theta_{3}^{f}+F_{3 ; 1}^{z} L_{3} \cos \theta_{3}^{f}=\frac{L_{3}}{2} m_{3} g_{z} \cos \theta_{3}^{f}-\frac{L_{3}}{2} m_{3} g_{y} \sin \theta_{3}^{f}
$$


for link $\mathcal{L}_{4}$, the force equilibrium equations are

$$
\begin{gathered}
F_{4 ; 2,3}^{y}+F_{4 ; 5}^{y}=m_{4} g_{y}, \\
F_{4 ; 2,3}^{z}+F_{4 ; 5}^{z}-F_{l l}=m_{4} g_{z},
\end{gathered}
$$

and the moment equilibrium equation is

$$
F_{4 ; 5}^{y} L_{4} \sin \theta_{4}^{f}-F_{4 ; 5}^{z} L_{4} \cos \theta_{4}^{f}+F_{l l} L_{4} \cos \theta_{4}^{f}=-\frac{L_{4}}{2} m_{4} g_{z} \cos \theta_{4}^{f}+\frac{L_{4}}{2} m_{4} g_{y} \sin \theta_{4}^{f},
$$

where $F_{l l}$ is the force from the unlock actuator which is assumed to work in the local $z$-direction; for link $\mathcal{L}_{5}$, the force equilibrium equations are

$$
\begin{aligned}
& F_{5 ; 4}^{y}+F_{5 ; 1}^{y}=m_{5} g_{y}, \\
& F_{5 ; 4}^{z}+F_{5 ; 1}^{z}=m_{5} g_{z},
\end{aligned}
$$

and the moment equilibrium equation is

$$
-F_{5 ; 1}^{y} L_{5} \sin \theta_{5}^{f}+F_{5 ; 1}^{z} L_{5} \cos \theta_{5}^{f}=\frac{L_{5}}{2} m_{5} g_{z} \cos \theta_{5}^{f}-\frac{L_{5}}{2} m_{5} g_{y} \sin \theta_{5}^{f} .
$$

From Equations (8b), the equilibrium equations for links $\mathcal{L}_{6}-\mathcal{L}_{9}$ can be formulated as follows: for link $\mathcal{L}_{6}$, the moment equilibrium equation is

$$
-F_{6 ; 7,8}^{y} L_{6} \sin \theta_{6}^{f}+F_{6 ; 7,8}^{z} L_{6} \cos \theta_{6}^{f}=\frac{L_{6}}{2} m_{6} g_{z} \cos \theta_{6}^{f}-\frac{L_{6}}{2} m_{6} g_{y} \sin \theta_{6}^{f} ;
$$

for link $\mathcal{L}_{7}$, the force equilibrium equations are

$$
\begin{aligned}
& F_{7 ; 6,8}^{y}+F_{7 ; 1}^{y}=m_{7} g_{y}, \\
& F_{7 ; 6,8}^{z}+F_{7 ; 1}^{z}=m_{7} g_{z},
\end{aligned}
$$

and the moment equilibrium equation is

$$
-F_{7 ; 1}^{y} L_{7} \sin \theta_{7}^{f}+F_{7 ; 1}^{z} L_{7} \cos \theta_{7}^{f}=\frac{L_{7}}{2} m_{7} g_{z} \cos \theta_{7}^{f}-\frac{L_{7}}{2} m_{7} g_{y} \sin \theta_{7}^{f}
$$


for link $\mathcal{L}_{8}$, the force equilibrium equations are

$$
\begin{gathered}
F_{8 ; 6,7}^{y}+F_{8 ; 9}^{y}=m_{8} g_{y}, \\
F_{8 ; 6,7}^{z}+F_{8 ; 9}^{z}-F_{\text {unlock }}=m_{8} g_{z},
\end{gathered}
$$

and the moment equilibrium equation is

$$
F_{8 ; 9}^{y} L_{8} \sin \theta_{8}^{f}-F_{8 ; 9}^{z} L_{8} \cos \theta_{8}^{f}+F_{\text {unlock }} L_{8} \cos \theta_{8}^{f}=-\frac{L_{8}}{2} m_{8} g_{z} \cos \theta_{8}^{f}+\frac{L_{8}}{2} m_{8} g_{y} \sin \theta_{8}^{f}
$$

and for link $\mathcal{L}_{9}$, the force equilibrium equations are

$$
\begin{aligned}
& F_{9 ; 8}^{y}+F_{9 ; 1}^{y}=m_{9} g_{y}, \\
& F_{9 ; 8}^{z}+F_{9 ; 1}^{z}=m_{9} g_{z},
\end{aligned}
$$

and the moment equilibrium equation is

$$
-F_{9 ; 1}^{y} L_{9} \sin \theta_{9}^{f}+F_{9 ; 1}^{z} L_{9} \cos \theta_{9}^{f}=\frac{L_{9}}{2} m_{9} g_{z} \cos \theta_{9}^{f}-\frac{L_{9}}{2} m_{9} g_{y} \sin \theta_{9}^{f} .
$$

The four force equilibrium equations for the upper sidestays (links $\mathcal{L}_{2}$ and $\mathcal{L}_{6}$ ) are not needed when forming the system of equations. This is because their inclusion introduces four new variables (the local planar components of the attachment point forces), so they only need to be included if attachment point force determination is required.

Along with the static equilibrium equations for the individual links, compatibility equations at the joints need to be included to ensure that there is no net force causing relative motion between the ends of adjoining links. This is achieved through inclusion of the constraint that the sum of the forces acting at the joint between two or more links must be zero. The compatibility equations at fore joints $C$ and $D$ (respectively) in the local $y$ and $z$ directions are therefore given by

$$
\begin{gathered}
F_{2 ; 3,4}^{y}+F_{3 ; 2,4}^{y}+F_{4 ; 2,3}^{y}=0, \\
F_{2 ; 3,4}^{z}+F_{3 ; 2,4}^{z}+F_{4 ; 2,3}^{z}=0, \\
F_{4 ; 5}^{y}+F_{5 ; 4}^{y}=0,
\end{gathered}
$$




$$
F_{4 ; 5}^{z}+F_{5 ; 4}^{z}=0,
$$

and the compatibility equations at aft joints $I$ and $J$ (respectively) in the local $y$ and $z$ directions by

$$
\begin{gathered}
F_{6 ; 7,8}^{y}+F_{7 ; 6,8}^{y}+F_{8 ; 6,7}^{y}=0, \\
F_{6 ; 7,8}^{z}+F_{7 ; 6,8}^{z}+F_{8 ; 6,7}^{z}=0, \\
F_{8 ; 9}^{y}+F_{9 ; 8}^{y}=0, \\
F_{8 ; 9}^{z}+F_{9 ; 8}^{z}=0 .
\end{gathered}
$$

(a)

(b)
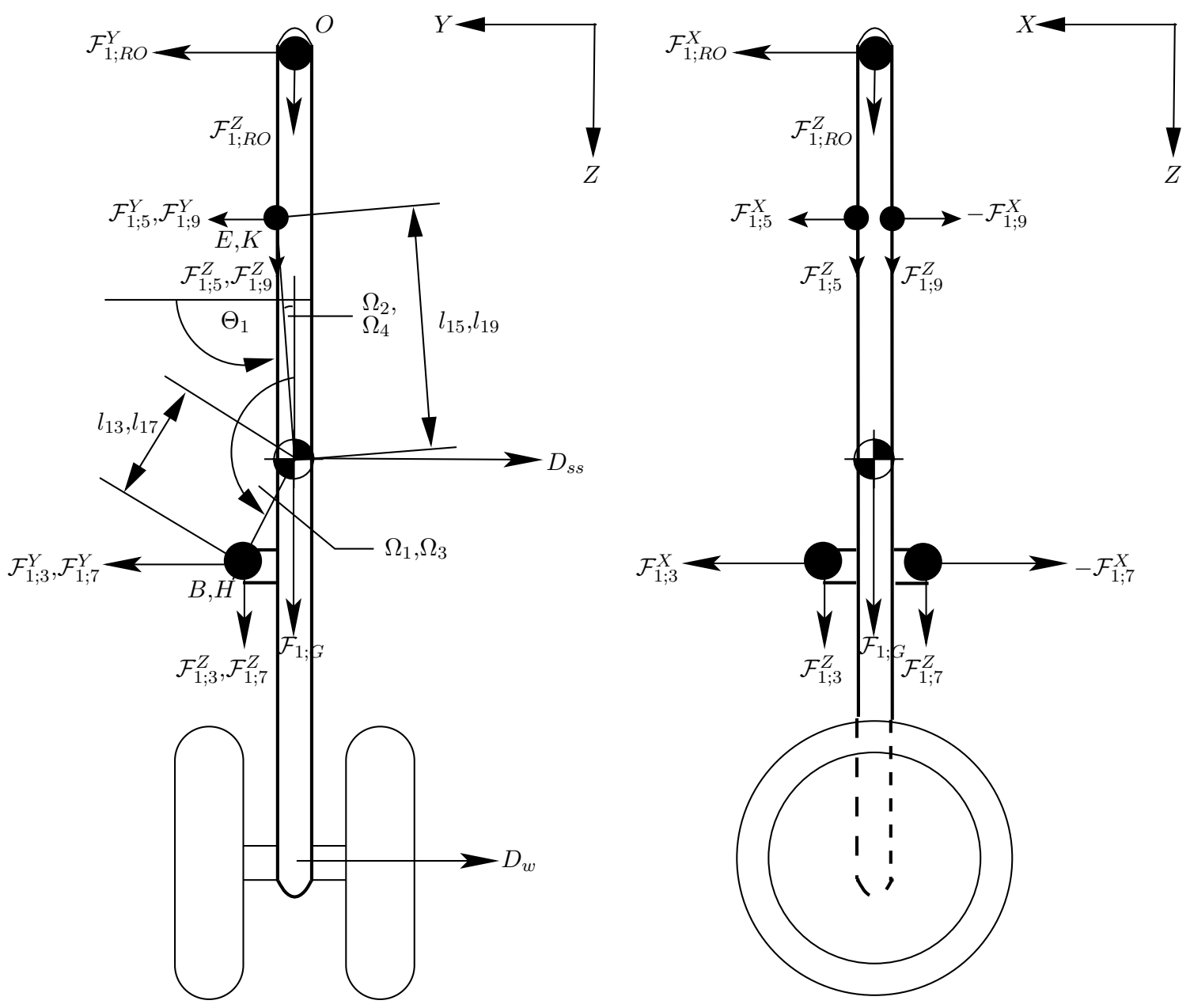

Figure 3: Free-body diagram of the DSS MLG shock strut viewed in global co-ordinates in the $(Y, Z)$-plane in which the gear retracts (a), and in the $(X, Z)$-plane $(\mathrm{b})$.

The compatibility equations at joints $B, E, H$ and $K$ require some forces to be calculated in global 
co-ordinates; they will be presented after the subsequent treatment of forces associated with the shock strut, link $\mathcal{L}_{1}$, which is more complicated than the force determination for the other links. Figure 3 shows the free-body diagram for the DSS MLG shock strut as viewed perpendicular to the retraction plane (along the global $X$-axis). The force and moment equilibrium equations for $\mathcal{L}_{1}$ can be constructed in a similar manner as those for the sidestays and locklinks, but the formulation becomes more complicated when considering the joint equilibrium equations between $\mathcal{L}_{1}$ and the adjoining links $\mathcal{L}_{3}, \mathcal{L}_{5}, \mathcal{L}_{7}$ and $\mathcal{L}_{9}$. These equations require the application of the inverse of the transformation matrices $T^{f}$ and $T^{a}$, to express the sidestay and locklink local forces in the global co-ordinate system in which the shock strut is considered. The joint equilibrium equation for the sidestay-shock strut and locklink-shock strut joints $(B, E, H$ and $K)$, respectively, are given by

$$
\begin{aligned}
& \mathcal{F}_{1 ; 3}=-\operatorname{inv}\left(T^{f}\right) F_{3 ; 1}^{f}, \\
& \mathcal{F}_{1 ; 5}=-\operatorname{inv}\left(T^{f}\right) F_{5 ; 1}^{f}, \\
& \mathcal{F}_{1 ; 7}=-\operatorname{inv}\left(T^{a}\right) F_{7 ; 1}^{a}, \\
& \mathcal{F}_{1 ; 9}=-\operatorname{inv}\left(T^{a}\right) F_{9 ; 1}^{a},
\end{aligned}
$$

where $i n v\left(T^{f}\right)$ and $i n v\left(T^{a}\right)$ are the inverse matrices of $T^{f}$ and $T^{a}$ and contain elements $t_{m, n}^{f}$ and $t_{m, n}^{a}$, respectively (where $m, n \in\{1,2,3\}$ ). Equations $(19 \mathrm{a}-\mathrm{d})$ can be expanded by multiplying out the right-hand side. Since the links are assumed to be rigid, only forces acting in the shock strut rotation plane influence the moment equilibrium of the link; therefore, expressions for the $X$-components of the global forces $\left(\mathcal{F}^{X}\right)$ have no effect on the mechanism and can be disregarded. Equation (20) describes eight of the internal structural forces shown in Figure 3:

$$
\begin{array}{ll}
\mathcal{F}_{1 ; 3}^{Y}=-\left(t_{2,1}^{f} F_{3 ; 1}^{x f}+t_{2,2}^{f} F_{3 ; 1}^{y f}+t_{2,3}^{f} F_{3 ; 1}^{z f}\right), & \mathcal{F}_{1 ; 3}^{Z}=-\left(t_{3,1}^{f} F_{3 ; 1}^{x f}+t_{3,2}^{f} F_{3 ; 1}^{y f}+t_{3,3}^{f} F_{3 ; 1}^{z f}\right), \\
\mathcal{F}_{1 ; 5}^{Y}=-\left(t_{2,1}^{f} F_{5 ; 1}^{x f}+t_{2,2}^{f} F_{5 ; 1}^{y f}+t_{2,3}^{f} F_{5 ; 1}^{z f}\right), & \mathcal{F}_{1 ; 5}^{Z}=-\left(t_{3,1}^{f} F_{5 ; 1}^{x f}+t_{3,2}^{f} F_{5 ; 1}^{y f}+t_{3,3}^{f} F_{5 ; 1}^{z f}\right), \\
\mathcal{F}_{1 ; 7}^{Y}=-\left(t_{2,1}^{a} F_{7 ; 1}^{x a}+t_{2,2}^{a} F_{7 ; 1}^{y a}+t_{2,3}^{a} F_{7 ; 1}^{z a}\right), & \mathcal{F}_{1 ; 7}^{Z}=-\left(t_{3,1}^{a} F_{7 ; 1}^{x a}+t_{3,2}^{a} F_{7 ; 1}^{y a}+t_{3,3}^{a} F_{7 ; 1}^{z a}\right), \\
\mathcal{F}_{1 ; 9}^{Y}=-\left(t_{2,1}^{a} F_{9 ; 1}^{x a}+t_{2,2}^{a} F_{9 ; 1}^{y a}+t_{2,3}^{a} F_{9 ; 1}^{z a}\right), & \mathcal{F}_{1 ; 9}^{Z}=-\left(t_{3,1}^{a} F_{9 ; 1}^{x a}+t_{3,2}^{a} F_{9 ; 1}^{y a}+t_{3,3}^{a} F_{9 ; 1}^{z a}\right) .
\end{array}
$$

The $y$ and $z$ components of $F_{3 ; 1}, F_{5 ; 1}, F_{7 ; 1}$ and $F_{9 ; 1}$ are described from the equilibrium equations of the fore and aft sidestays and locklinks, but the out-of-plane components in the local $x$-direction $\left(F_{3 ; 1}^{x f}, F_{5 ; 1}^{x f}, F_{7 ; 1}^{x a}\right.$ and $\left.F_{9 ; 1}^{x a}\right)$ require calculating explicitly. These force components are calculated by considering the sidestay 
planes in isolation.

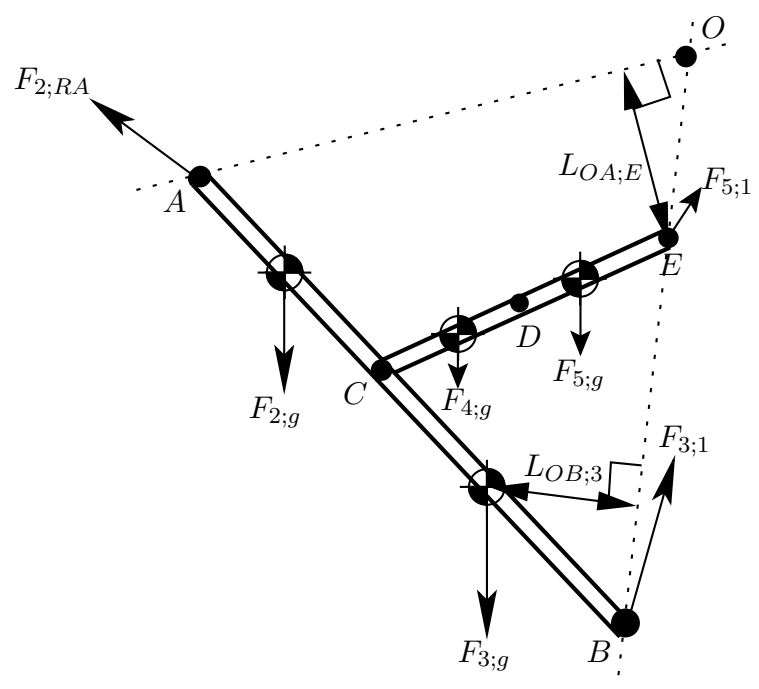

Figure 4: Free-body diagram of the fore sidestay plane.

The free-body diagram used to obtain $F_{3 ; 1}^{x f}$ and $F_{5 ; 1}^{x f}$ is shown in Figure 4 . There are three unknown forces, acting at points $A, B$ and $E$, which need to be determined before the equilibrium equation for the shock strut can be considered. The $x$-components of the forces are assigned to act in a positive direction. A similar free-body diagram can be constructed for the aft sidestay-locklink plane, which allows $F_{7 ; 1}^{x a}$ and $F_{9 ; 1}^{x a}$ to be calculated in exactly the same way. The $x$-components of the six unknown forces, $\left(F_{3 ; 1}^{f}, F_{5 ; 1}^{f}, F_{2 ; R A}^{f}\right.$, $F_{7 ; 1}^{a}, F_{9 ; 1}^{a}$ and $\left.F_{6 ; R G}^{a}\right)$ can be obtained by resolving forces perpendicular to each sidestay plane and applying moment equilibrium about four axes $(\overline{O A}, \overline{O B}, \overline{O G}$ and $\overline{O H})$.

The reaction force $x$-components $F_{2 ; R A}^{x f}$ and $F_{6 ; R G}^{x a}$ can be calculated directly as

$$
\begin{aligned}
F_{2 ; R A}^{x f}=\frac{-1}{L_{O B ; A}} & \left(F_{2 ; g}^{x f} L_{O B ; 2}+F_{3 ; g}^{x f} L_{O B ; 3}\right. \\
& \left.+F_{4 ; g}^{x f} L_{O B ; 4}+F_{5 ; g}^{x f} L_{O B ; 5}\right) \\
F_{6 ; R G}^{x a}=\frac{-1}{L_{O H ; G}} \quad & \left(F_{6 ; g}^{x a} L_{O H ; 6}+F_{7 ; g}^{x a} L_{O H ; 7}\right. \\
& \left.+F_{8 ; g}^{x a} L_{O H ; 8}+F_{9 ; g}^{x a} L_{O H ; 9}\right)
\end{aligned}
$$

Here, as before, $F_{2 ; R A}^{x f}$ is the fore plane $x$-component of force $F_{2 ; R A}^{f}$ in the fore plane co-ordinates, and $F_{2 ; R G}^{x a}$ is the equivalent force in the aft plane. The generalised moment arm $L_{O B ; *}$ is the shortest length from axis $\overline{O B}$ to point $*$. Similarly, the generalised moment arm $L_{O H ; *}$ is the shortest length from axis $\overline{O H}$ to point $*$ for the aft plane's equations. The example shown in Figure 4 depicts the moment arm $L_{O B ; 3}$ which is the moment arm of the lower sidestay's weight $\left(F_{3 ; g}^{f}\right)$ about axis $\overline{O B}$. After obtaining $F_{2 ; R A}^{x f}$, the following two expressions can be solved simultaneously to obtain $F_{3 ; 1}^{x f}$ and $F_{5 ; 1}^{x f}$ : 


$$
\begin{gathered}
F_{2 ; R A}^{x f}+F_{2 ; g}^{x f}+F_{3 ; g}^{x f}+F_{4 ; g}^{x f}+F_{5 ; g}^{x f}+F_{3 ; 1}^{x f}+F_{5 ; 1}^{x f}=0, \\
F_{2 ; g}^{x f} L_{O A ; 2}+F_{3 ; g}^{x f} L_{O A ; 3}+F_{4 ; g}^{x f} L_{O A ; 4} \\
\quad+F_{5 ; g}^{x f} L_{O A ; 5}+F_{3 ; 1}^{x f} L_{O A ; B}+F_{5 ; 1}^{x f} L_{O A ; E}=0 .
\end{gathered}
$$

In Equation (22b) the generalised moment arm $L_{O A ; *}$ is the shortest length from axis $\overline{O A}$ to point $*$. Figure 4 also depicts the distance $L_{O A ; E}$; this is the moment arm of the internal force $F_{5 ; 1}$ (which acts at joint $E$ in the MLG) about axis $\overline{O A}$. The equivalent equations, for the aft plane, to those given by Equation (22) are:

$$
\begin{gathered}
F_{6 ; R G}^{x a}+F_{6 ; g}^{x a}+F_{7 ; g}^{x a}+F_{8 ; g}^{x a}+F_{9 ; g}^{x a}+F_{7 ; 1}^{x a}+F_{9 ; 1}^{x a}=0, \\
F_{6 ; g}^{x a} L_{O H ; 6}+F_{7 ; g}^{x a} L_{O G ; 7}+F_{8 ; g}^{x a} L_{O G ; 8} \\
+F_{9 ; 9}^{x a} L_{O G ; 9}+F_{7 ; 1}^{x a} L_{O G ; H}+F_{9 ; 1}^{x a} L_{O G ; K}=0 .
\end{gathered}
$$

The moment equilibrium for the shock strut, along with the compatibility equations for joints $B, E, H$ and $K$, can now be formulated in terms of the global forces shown in Figure 3 (expressed mathematically in Equation (20)). The fore and aft plane force $x$-components $\left(F_{3 ; 1}^{x f}, F_{5 ; 1}^{x f}, F_{7 ; 1}^{x a}\right.$ and $\left.F_{9 ; 1}^{x a}\right)$ are calculated from Equations (22) and (23), and all other components are solved by the continuation algorithm as system states. The moment equilibrium equation for $\mathcal{L}_{1}$ is

$$
\begin{array}{r} 
\\
\mathcal{F}_{1 ; 3}^{y}\left(\frac{L_{1}}{2} \sin \Theta_{1}-l_{13} \sin \left(\Theta_{1}+\Omega_{1}\right)\right)+\mathcal{F}_{1 ; 3}^{z}\left(l_{13} \cos \left(\Theta_{1}+\Omega_{1}\right)-\frac{L_{1}}{2} \cos \Theta_{1}\right) \\
+\mathcal{F}_{1 ; 5}^{y}\left(\frac{L_{1}}{2} \sin \Theta_{1}-l_{15} \sin \left(\Theta_{1}-\Omega_{2}\right)\right)+\mathcal{F}_{1 ; 5}^{z}\left(l_{15} \cos \left(\Theta_{1}-\Omega_{2}\right)-\frac{L_{1}}{2} \cos \Theta_{1}\right) \\
+\mathcal{F}_{1 ; 7}^{y}\left(\frac{L_{1}}{2} \sin \Theta_{1}-l_{17} \sin \left(\Theta_{1}+\Omega_{3}\right)\right)+\mathcal{F}_{1 ; 7}^{z}\left(l_{17} \cos \left(\Theta_{1}+\Omega_{3}\right)-\frac{L_{1}}{2} \cos \Theta_{1}\right) \\
+\mathcal{F}_{1 ; 9}^{y}\left(\frac{L_{1}}{2} \sin \Theta_{1}-l_{19} \sin \left(\Theta_{1}-\Omega_{4}\right)\right)+\mathcal{F}_{1 ; 9}^{z}\left(l_{19} \cos \left(\Theta_{1}-\Omega_{4}\right)-\frac{L_{1}}{2} \cos \Theta_{1}\right) \\
=\left(\frac{m_{1}}{2} G_{z}+m_{\text {wheel }}\right) L_{1} \cos \Theta_{1}+\mathcal{F}_{\text {act }}^{z}\left(l_{1 a c t} \cos \left(\Theta_{1}+\Omega_{5}\right)\right)-\mathcal{F}_{\text {act }}^{y}\left(l_{1 a c t} \sin \left(\Theta_{1}+\Omega_{5}\right)\right)+M_{D}-M .
\end{array}
$$

Here $M$ is the retraction actuator moment parameter, defined to act about the shock-strut attachment point $O ; F_{a c t}$ is the retraction actuator force parameter, which acts between the shock-strut and airframe during retraction; $M_{D}$ is the drag-induced moment (set to zero in this work); length $l_{1 a c t}$ is the distance from the shock strut cg to the adjoining retraction actuator, and $\Omega_{5}$ is the angle that the length $l_{1 a c t}$ makes with the 
shock strut centreline (in local co-ordinates). Angles $\Omega_{1-4}$ are as depicted in Figure 3, and all other entities follow the defined naming conventions for force, angle and link positional states.

The compatibility equations for joints associated with the shock strut are:

$$
\begin{aligned}
& \mathcal{F}_{1 ; 3}^{y}+t_{2,2}^{f} F_{3 ; 1}^{y}+t_{2,3}^{f} F_{3 ; 1}^{z}=-t_{2,1}^{f} F_{3 ; 1}^{x}, \\
& \mathcal{F}_{1 ; 3}^{z}+t_{3,2}^{f} F_{3 ; 1}^{y}+t_{3,3}^{f} F_{3 ; 1}^{z}=-t_{3,1}^{f} F_{3 ; 1}^{x}, \\
& \mathcal{F}_{1 ; 5}^{y}+t_{2,2}^{f} F_{5 ; 1}^{y}+t_{2,3}^{f} F_{5 ; 1}^{z}=-t_{2,1}^{f} F_{5 ; 1}^{x}, \\
& \mathcal{F}_{1 ; 5}^{z}+t_{3,2}^{f} F_{5 ; 1}^{y}+t_{3,3}^{f} F_{5 ; 1}^{z}=-t_{3,1}^{f} F_{5 ; 1}^{x}, \\
& \mathcal{F}_{1 ; 7}^{y}+t_{2,2}^{a} F_{7 ; 1}^{y}+t_{2,3}^{a} F_{7 ; 1}^{z}=-t_{2,1}^{a} F_{7 ; 1}^{x}, \\
& \mathcal{F}_{1 ; 7}^{z}+t_{3,2}^{a} F_{7 ; 1}^{y}+t_{3,3}^{a} F_{7 ; 1}^{z}=-t_{3,1}^{a} F_{7 ; 1}^{x}, \\
& \mathcal{F}_{1 ; 9}^{y}+t_{2,2}^{a} F_{9 ; 1}^{y}+t_{2,3}^{a} F_{9 ; 1}^{z}=-t_{2,1}^{a} F_{9 ; 1}^{x}, \\
& \mathcal{F}_{1 ; 9}^{z}+t_{3,2}^{a} F_{9 ; 1}^{y}+t_{3,3}^{a} F_{9 ; 1}^{z}=-t_{3,1}^{a} F_{9 ; 1}^{x} .
\end{aligned}
$$

The forces on the left-hand sides of Equations (25a-h) are the unknowns, to be determined by the continuation algorithm. The force values on the right-hand side are calculated explicitly from Equations (21)-(23).

As with the cases of the upper sidestays (links $\mathcal{L}_{2}$ and $\mathcal{L}_{6}$ ), the force equilibrium equations for the shock strut only need to be included if the force at its attachment point is required. For the purposes of this work, the attachment point forces are not needed so the force equilibrium equations for $\mathcal{L}_{1}$ are not included in the model.

\section{Matrix Formulation}

To simplify model implementation, specifically the requirement for determining an initial continuation starting point, the 37 internal force/moment equilibrium equations presented thus far (i.e. Equations (9)-(18) and (25)-(24)) were combined into a matrix form such that

$$
A \bar{F}-\bar{B}=0 .
$$

Formulating the landing gear force-balance equations in this way eases calculation of the initial conditions, by computing $A^{-1} \bar{B}$ (provided $A^{-1}$ is nonsingular, which is the case here). The generic formulation of Equation (26) is the same as that used for the single sidestay MLG model, however the matrix $A$ and vectors $\bar{F}$ and $\bar{B}$ are significantly larger than before. For the single sidestay MLG model, ${ }^{15} A$ is a $(19 \times 19)$ matrix, 
containing the terms from the equilibrium equations which are multiplied by the vector of internal forces $\bar{F}$. The addition of an extra sidestay causes $A$ to practically double in size to a $(37 \times 37)$ matrix. Note that $A$ does not double in size exactly, because there is only one shock strut (described by one row in $A$ ) - all other elements (i.e. those for the sidestays and locklinks) are doubled when comparing the DSS MLG model to the single-sidestay MLG model.

Rather than presenting matrix $A$ as a single, sparse matrix, the elements within $A$ can be grouped such that the force coefficients from the global, fore and aft constraint equations become sub-matrices in $A$. In this way, Equation (26) can be written as

$$
\left[\begin{array}{cccccc} 
& & & 0 & \ldots & 0 \\
& {\left[A^{f}\right]} & & \vdots & \ddots & \vdots \\
& & & 0 & \ldots & 0 \\
0 & \ldots & 0 & & & \\
\vdots & \ddots & \vdots & & {\left[A^{a}\right]} & \\
0 & \ldots & 0 & & & \\
C_{1} & \ldots & \ldots & \ldots & \ldots & C_{37}
\end{array}\right]\left[\begin{array}{c}
\bar{F}^{f} \\
\bar{F}^{a}
\end{array}\right]-\left[\begin{array}{c}
\bar{B}^{f} \\
\bar{B}^{a} \\
B^{\mathcal{L}_{1}}
\end{array}\right]=0 .
$$

The matrix $A$ in Equation (26) is formed as two matrices $A^{f}$ and $A^{a}$, which describe the forces in the sidestay-locklink fore and aft planes, along with the vector $\bar{C}$, which describes the shock strut forces in the global co-ordinate system. $A^{f}$ and $A^{a}$ are $(18 \times 19)$ and $(18 \times 18)$ matrices, respectively, whilst the row vector $\bar{C}=\left\{C_{1} \ldots C_{37}\right\}$ is mathematically responsible for linking the fore and aft planes. It contains mainly zeros, with eight non-zero entries which describe the moment equilibrium of the shock strut in terms of forces applied by the fore and aft plane. Vector $\bar{F}$ in Equation (26) is defined in terms of two separate vectors, $\bar{F}^{f}$ and $\bar{F}^{a}$, for the fore- and aft-plane force elements, whilst vector $\bar{B}$ contains the corresponding non-forcecoefficient terms. All the matrices and vectors in Equation 27 are given in Appendix I. Equation 27, together with the geometric constraints of Equations 6, constitute the DSS MLG model. 


\section{E. Addition of structural flexibilities}

By using the internal structural forces, it is straightforward to include an element of structural flexibility within the model. The DSS MLG retraction and deployment is highly sensitive to geometric deflections, especially around the downlock point. In fact, for a fully rigid landing gear, the downlock solution becomes unobtainable if one of the sidestay attachment points is moved from the undeflected geometry. For such cases, some aspect of the DSS MLG geometry must change in order for the gear to reach the downlock point: in the real system, this occurs through a combination of the shock strut bending, whilst the sidestays compress or extend on alternate sides. For ease of initial implementation here, only the sidestay compression/extension is modelled, by treating the sidestay length parameters as highly stiff linear springs. The formulation described here is applicable to all sidestay links $\left(\mathcal{L}_{2}, \mathcal{L}_{3}, \mathcal{L}_{6}\right.$ and $\left.\mathcal{L}_{7}\right)$.

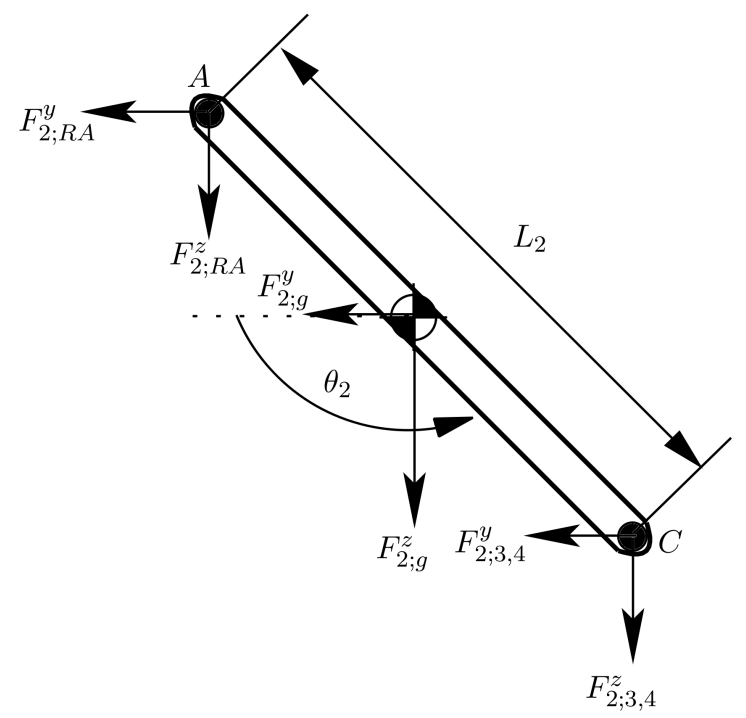

Figure 5: Free-Body Diagram of the fore upper sidestay link $\mathcal{L}_{2}$.

Figure 5 shows the upper fore sidestay link with internal forces from the adjoining links acting at either end. The link weight acts at the link centre of gravity. All forces are depicted to act in the local co-ordinate positive $y$ - and $z$-directions. The forces at either end of the link can be considered, with the link weight added onto the $z$-component of the force, and resolved into components axially and perpendicularly to the link. The change in link length can then be calculated by dividing the axial force component with the link stiffness, $k_{i}$. The flexible link's length is therefore described in the general case by

$$
L_{i}=\frac{1}{k_{i}}\left(\left(F_{i ; *}^{y}+m_{i} g_{y}\right) \cos \theta_{i}+\left(F_{i ; *}^{z}+m_{i} g_{z}\right) \sin \theta_{i}\right)+L 0_{i}
$$

where $L 0_{i}$ is the unstrained length of link $\mathcal{L}_{i}$ (i.e. the length when there are no forces acting axially on the bar). For the case of $\mathcal{L}_{2}$ (as depicted in Figure 5) Equation (28) becomes: 


$$
L_{2}=\frac{1}{k_{2}}\left(\left(F_{2 ; 3,4}^{y}+m_{2} g_{y}\right) \cos \theta_{2}+\left(F_{2 ; 3,4}^{z}+m_{2} g_{z}\right) \sin \theta_{2}\right)+L 0_{2},
$$

where $m_{2} g_{y}=F_{2 ; g}^{y}$ and $m_{2} g_{z}=F_{2 ; g}^{z}$. The linear relation between force and displacement used by this model is applicable for an isotropic material undergoing small (i.e. elastic) deflections; as most landing gears are constructed with metal sidestays, and the deflections considered in the results in Section IV are much less than $1 \%$ of the sidestay link lengths, these assumptions are reasonable for a real DSS MLG. The stiffness is also assumed to be unaffected by the position along the link where the force is applied, which is a reasonable assumption as only small axial deflections are considered.

\section{F. Retraction Actuator Parameterisation}

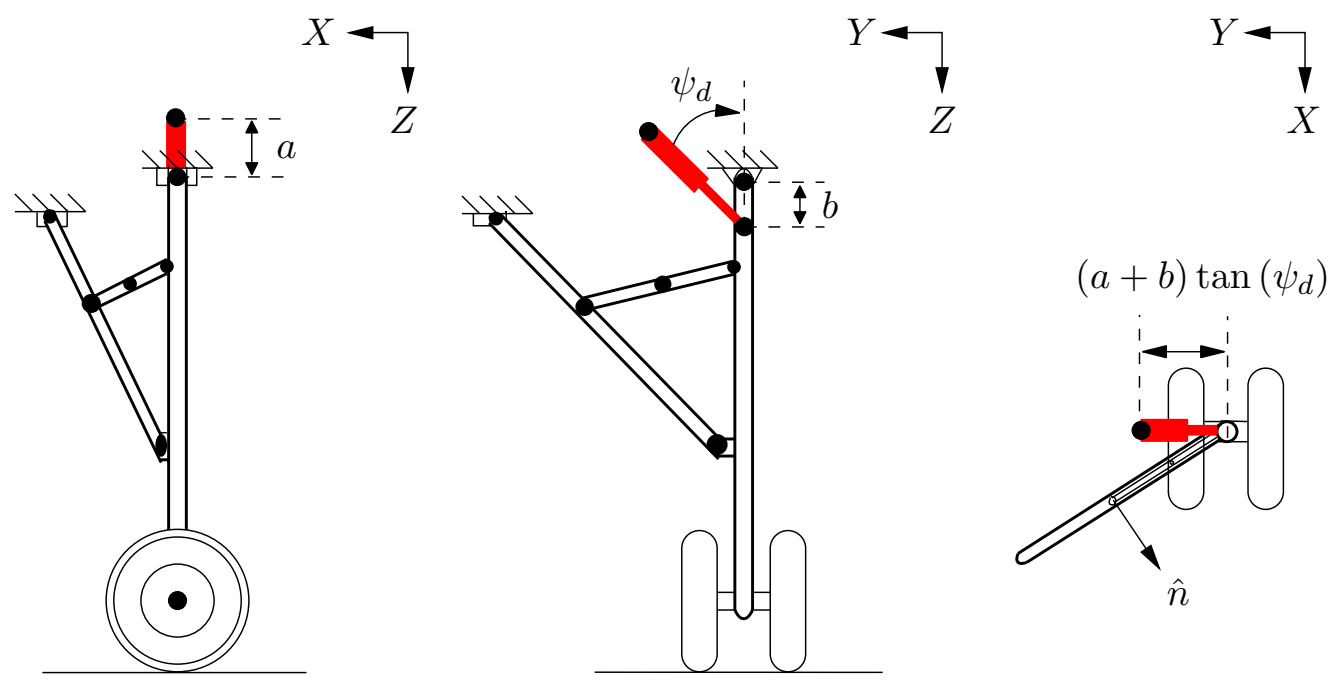

Figure 6: Retraction actuator parameterisation diagram.

Figure 6 shows how the retraction actuator position is parameterised within the model. Three parameters are used to describe the actuator position: length $a$ denotes the vertical distance between the shock strut rotation point and the actuator attachment point on the aircraft body; length $b$ is the distance between the shock strut rotation point and the actuator attachment point on the shock strut; and angle $\psi_{d}$ is the angle made in the deployed position between the actuator and the shock strut centreline. It should be noted that, whilst it would be possible to parameterise the actuator position in terms of three length parameters $(a, b$ and $\left.c=(a+b) \tan \psi_{d}\right)$ rather than two lengths and one angle, the three length parameters would not be independent so that distinguishing between parameter effects would be more difficult.

The retraction actuator is positioned in the plane in which the shock strut retracts, as any out of plane actuator components would not contribute to retracting the landing gear in this rigid model. The actuator 
was also assumed to be attached to the shock strut centreline, something which is not necessarily the case for all real landing gears. The parameter $a$ was fixed for the following results presented in this paper, and chosen to be equal to $10 \%$ of the overall landing gear length.

\section{Numerical Continuation of Sidestay Angle}

The formulation flexibility allows the sidestay attachment points in the DSS MLG model to be parameterised in terms of a fore and aft attachment point angle $\left(\alpha^{f}\right.$ and $\alpha^{a}$ respectively; see Figure 1), which can then be continued numerically to allow different geometries to be considered. Specifying two angles to describe the two sidestay attachment point positions enables consideration of both symmetric $\left(\alpha^{f}=\alpha^{a}\right)$ and asymmetric $\left(\alpha^{f} \neq \alpha^{a}\right)$ geometries. The following subsections consider several asymmetric geometry cases.

\section{A. Comparison of Single-Sidestay and Dual-Sidestay MLG models}

To ensure confidence in the DSS model formulation, the retraction cycle for the DSS MLG is compared to that of the single-sidestay MLG presented previously. ${ }^{15}$ By setting $\alpha^{f}=-\alpha^{a}$ and making the sidestay and locklink masses in the DSS model half the value of the sidestay and locklink masses in the single sidestay model (such that the total mass of both gears is the same), the resulting DSS MLG is equivalent to a single sidestay MLG.

Figure 7 shows the retraction actuator force $F$ in the DSS MLG model as a function of the shock strut angle $\Theta_{1}$ and the deployed actuator angle $\psi_{d}$. The case presented is that when the two sidestays are aligned with one another, i.e. $\alpha^{a}=-\alpha^{f}=-35^{\circ}$. The light curves of panels (a) and (b) show the response of the single-sidestay MLG, which is matched practically exactly by the equivalent DSS MLG retraction curves in black. The only noticeable difference between the single and dual sidestay retraction results is that the DSS retraction curves stop at a retraction angle of $\Theta_{1} \approx 10^{\circ}$, whereas the single sidestay MLG model retracts to $\Theta_{1}=0^{\circ}$. The reason for this is that the DSS MLG is not exactly equivalent (geometrically) to the single sidestay MLG. Because the model was initialised by numerically continuing the sidestays together from a symmetric geometry, the final value for the continued angle is obtained once the continuation has reached a parameter value one step beyond the specified parameter end point. This means that, whilst the two sidestay planes in the DSS model are approximately the same, there is a very small angle (about $0.05^{\circ}$ ) between the fore and aft planes. This small difference is thought to cause the gear to lock at the point when the sidestay plane normal vectors reach the vertical plane (i.e. the global $(Y, Z)$-plane), presenting numerical difficulties in tracing solutions beyond this point. These numerical difficulties are a direct consequence of the overdetermined nature of a DSS mechanism. Physically, this means that, as the fully retracted position (where the two locklinks are aligned) is approached, the two 

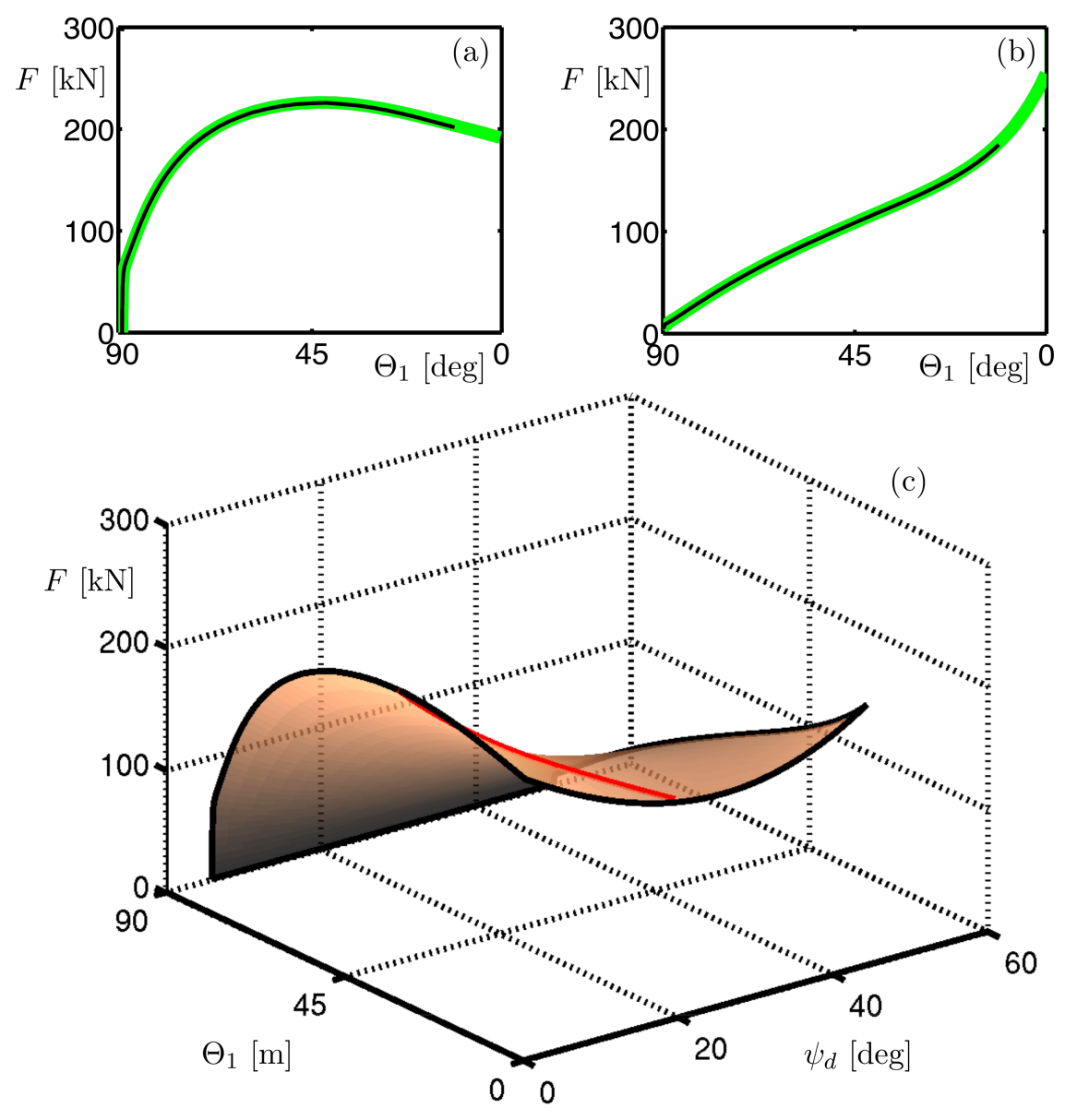

Figure 7: Comparison between dual-sidestay and single-sidestay landing gear retraction, for actuator angle values of: $\psi_{d}=6^{\circ}(\mathrm{a}), \psi_{d}=50^{\circ}(\mathrm{b})$, and $\psi_{d}$ between $6^{\circ}$ and $50^{\circ}(\mathrm{c})$. The black curves in panels (a) and (b) are for the DSS MLG with $\alpha^{a}=\alpha^{f}=35^{\circ}$, and the light curves are for equivalent results from the single-sidestay model. The angles $\Theta_{1}$ and $\psi_{d}$ are the shock strut angle and initial retraction actuator angle respectively, with force $F=\sqrt{\mathcal{F}_{\text {act }}^{y^{2}}+\mathcal{F}_{\text {act }}^{z^{2}}}$ indicating the retraction actuator force magnitude in the retraction plane.

sidestays may increasingly work against each other (creating large forces) in the presence of numerical inaccuracies. In this situation, the solution may be difficult to follow numerically.

Figure 7(c) shows the surface of steady-state solutions of the DSS MLG as a function of both the shock strut or retraction angle $\Theta_{1}$, and the deployed actuator angle $\psi_{d}$. On the surface is a locus of local maxima, present for a range of $\psi_{d}$. At low values of the deployed actuator angle $\psi_{d}$, the retraction profile is qualitatively similar to the case shown in Figure 7(a). The local maximum point occurs increasingly later in the retraction cycle (i.e. at lower $\Theta_{1}$ values) as $\psi_{d}$ is increased. As $\psi_{d}$ increases past the value where the local maximum occurs in the retracted position, there is a qualitative change in the retraction response. For these high values of $\psi_{d}$, the retraction profile is qualitatively similar to that shown in Figure 7(b).

The overall behaviour shows very good qualitatative and quantitative agreement with an equivalent 
surface for a single-sidestay MLG in previous work. ${ }^{15}$

\section{B. Numerical Continuation of Aft Sidestay Angle}

From the single-sidestay configuration, the aft sidestay attachment point can be continued through changing the aft sidestay angle $\alpha^{a}$, while keeping $\alpha^{f}$ fixed at $35^{\circ}$. The resulting qualitative effect of the change in geometry on the retraction surface is shown in Figure 8.
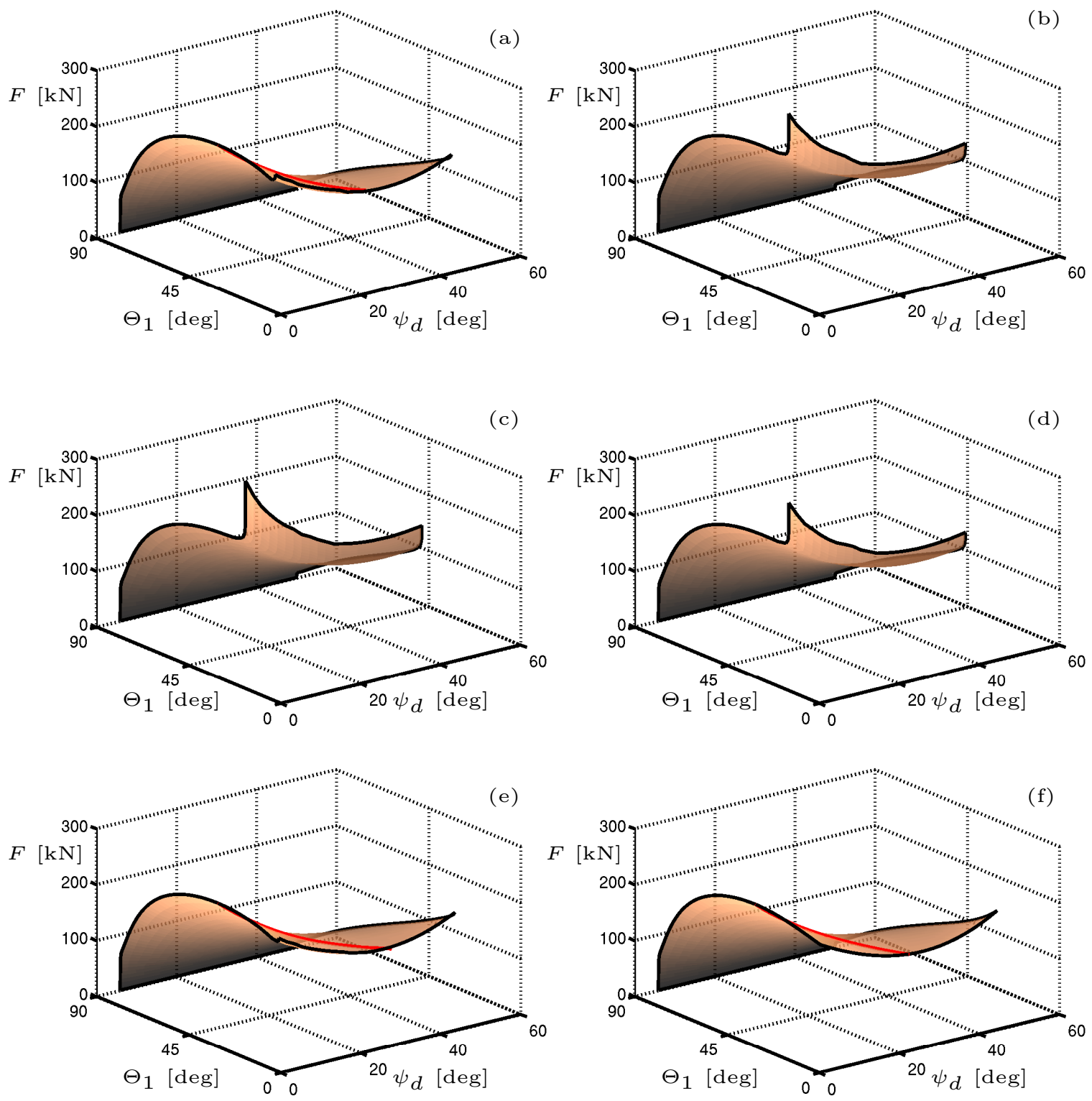

Figure 8: Qualitative effect of increasing the angle between the two sidestays in the DSS MLG model, with retraction surfaces shown for aft sidestay angles of: $\alpha^{a}=-20^{\circ}(\mathrm{a}), \alpha^{a}=-10^{\circ}(\mathrm{b}), \alpha^{a}=0^{\circ}$ (c), $\alpha^{a}=10^{\circ}$ (d), $\alpha^{a}=20^{\circ}(\mathrm{e}), \alpha^{a}=35^{\circ}$ (f).

For the retraction surfaces shown in Figure 8, the fore sidestay plane angle $\alpha^{f}$ remains fixed whilst the 
aft sidestay swings out through $10^{\circ}$ intervals from $\alpha^{a}=-20^{\circ}$ to $\alpha^{a}=20^{\circ}$ (panels (a) to (e)), before the symmetric case $\left(\alpha^{a}=\alpha^{f}=35^{\circ}\right)$ is presented in the final panel (f).

There appears to be a good qualitative match between positive and negative $\alpha^{a}$ values. Considering the pairs of surfaces in Figures 8(a) and (e), and (b) and (d), the locus of local maxima (curve on surface) disappears when $\left|\alpha^{a}\right|$ decreases from $20^{\circ}$ to $10^{\circ}$, yet the surfaces show no significant differences when comparing positive and negative $\alpha^{a}$ values. The reasons for this behaviour are due to the role the sidestay geometry plays in the retraction of the MLG, and are discussed below.

When considering the causes of the observed actuator force variation with retraction angle, the aft sidestay and locklink weight is the physical aspect of the sidestay plane which works against the retraction actuator force. This weight is transferred to the shock strut at two points along its length, and these points remain constant irrespective of the aft sidestay plane angle $\alpha^{a}$. Because the shock strut is rigid, the weight component opposing the shock strut movement for any single positive $\alpha^{a}$ is identical to the weight component for the negative of that same value of $\alpha^{a}$. Furthermore, the geometric symmetry of a MLG for positive and negative values of a given $\alpha^{a}$ results in the observed similarities between Figures 7(c) and 8(f) $\left(\alpha^{a}=\mp 35^{\circ}\right)$, Figures 8(a) and (e) $\left(\alpha^{a}=\mp 20^{\circ}\right)$, and Figure 8(b) and (d) $\left(\alpha^{a}=\mp 10^{\circ}\right)$. The noticeable differences between Figures 8(a) and (e) arise because the sidestay angle was continued to the exact value, but the continuation run only stops once that parameter value has been exceeded (as explained in Section IIIA).

The other effect the sidestay and locklink geometry has on the retraction loads relates to the MLG as a purely geometric mechanism. For the MLG to fully retract, all sidestay and locklink links must be able to rotate about one another at a fixed distance (as the mechanism is still rigid for this analysis). If, at a certain point in the retraction cycle, the links are no longer able to rotate about one another without violating their length constraints, the mechanism locks and becomes a structure. Increasing the externally applied force (in this case from the retraction actuator) merely causes the internal forces to redistribute, but no movement in the mechanism occurs. It is this mechanism lockup that causes the retraction surface to become vertical for low retraction angles as $\left|\alpha^{a}\right| \rightarrow 0$.

The reason this lockup is a function of $\alpha^{a}$ is that the sidestay plane angle affects the motion of the sidestays and locklinks throughout the retraction: for small absolute values of $\alpha^{a}$, the sidestays and locklinks rotate in their plane more than they do for higher values of $\alpha^{a}$. This is shown in Figure 9, which depicts how the relation between $\theta_{8}^{a}$ and $\Theta_{1}$ changes as the aft sidestay plane is moved from the single sidestay configuration $\left(\alpha^{a}=-35^{\circ}\right)$ through to the symmetric DSS configuration $\left(\alpha^{a}=35^{\circ}\right)$. For the single sidestay configuration, there is a corresponding $\theta_{8}^{a}$ value for the whole range of retraction angles considered $\left(90^{\circ}\right.$ down to $\left.10^{\circ}\right)$. As the aft sidestay plane moves away from the fore sidestay plane, there is a qualitative change in the relation between $\theta_{8}^{a}$ and $\Theta_{1}$. When $\alpha^{a}=-20^{\circ}$, Figure $9($ a) shows the relation becomes more parabolic, appearing to 

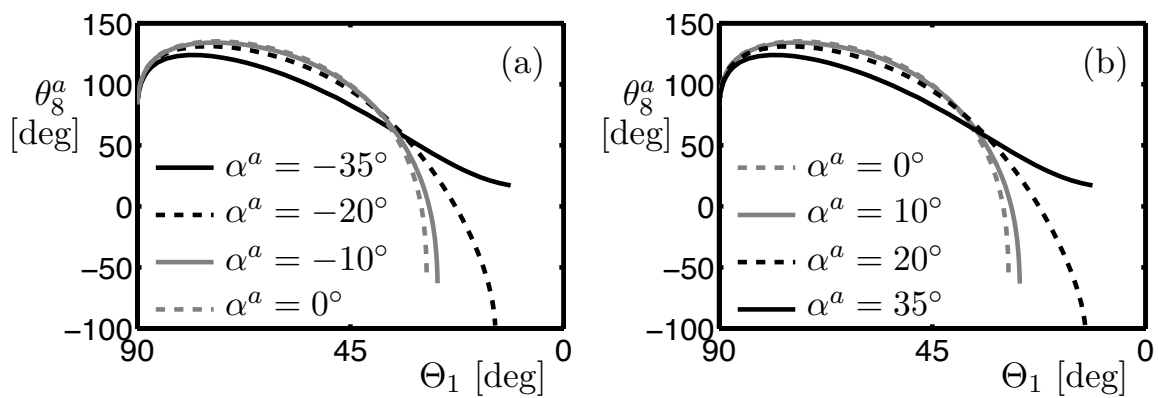

Figure 9: Variation in aft locklink angle $\theta_{8}^{a}$ as a function of retraction angle $\Theta_{1}$ for different values of aft sidestay plane angle $\alpha^{a}$. Panel (a) considers the cases where $\alpha^{a} \leq 0$, whilst panel (b) presents the cases when $\alpha^{a} \geq 0$.

approach a $\Theta_{1}$ asymptotic value. Furthermore, the value of $\theta_{8}^{a}$ passes through zero for $\alpha^{a} \geq-20^{\circ}$, showing that this locklink is rotating more as the sidestay plane angle is increased towards zero.

As the sidestay plane angle increases further, the landing gear mechanism begins to lock up at lower values of retraction angle $\Theta_{1}$. This is shown by the increased gradient over the latter part of the curves in Figure $9(\mathrm{a})$. The easiest case to consider is that where $\alpha^{a}=0^{\circ}$, as the aft sidestay plane remains fixed in space for this case (i.e. it does not rotate as the gear retracts). The crucial joint to consider is the aft joint $I$, joining the aft sidestays to the locklinks; see Figure 1. As the mechanism is rigid, this link must trace out a circular path about the aft sidestay attachment point $G$. At the same time, the distance between the ends of the two locklinks (joints $I$ and $K$ ) cannot exceed the lengths of both locklinks $\left(L_{8}\right.$ and $\left.L_{9}\right)$. The mechanism therefore locks up at the point where these two conditions can no longer be satisfied, because the locklinks are being stretched by the rest of the mechanism.

Figure 9(b) presents equivalent results to those in Figure $9(\mathrm{a})$ for the cases when $\alpha^{a} \geq 0$. The results are identical (within the error tolerances of the continuation) to those discussed for $\alpha^{a} \leq 0$. This is an expected result as there is no difference in the geometric relations in the landing gear (such as the presented relation between $\theta_{8}^{a}$ and $\left.\Theta_{1}\right)$ for $\alpha^{a}=+x^{\circ}$ or $\alpha^{a}=-x^{\circ}$.

\section{Downlock Sensitivity Study}

The DSS MLG mechanism will enable the gear to move between retracted and deployed states provided the geometry is in its nominal position, i.e. no attachment points have moved nor any lengths changed. This situation is unrealistic in a real landing gear system, because under flight conditions the wing, and hence the MLG attachment points, will deflect. If the landing gear is rigid, any asymmetric deflections (where one attachment point moves relative to the other) mean the downlock solution for the gear no longer exists. Since real structures and mechanisms are not rigid, the downlock solution for a real landing gear may still 
be obtainable, but some elements within the structure would also need to deflect in order for the downlock point to be reached. This translates to a force barrier that needs to be overcome for successful locking.

In order to mimic the asymmetric downlock case, the following numerical continuation results are started with the landing gear in a semi-locked position. The aft plane is downlocked, but the fore plane locklinks are left unlocked. A deflection to the aft attachment point is introduced by defining a length parameter along the vector intersecting points $G$ and $H$, and then numerically continuing this parameter until the desired deflection is obtained. From this solution, the landing gear is treated as being half-downlocked by fixing the rotational state $\Theta_{1}$. Because one state has been fixed, one fixed parameter is required to vary for the problem to be well posed (otherwise it would be over-constrained). The real landing gear would be held in place by the locklinks pressing against one another, so an artificial resistance force is added that acts between the two aft-plane locklinks. The variation of this force effectively keeps the MLG in equilibrium throughout the subsequent downlock analysis continuation runs.

From the semi-locked state, the unlock force in the fore plane is used as a continuation parameter. In order for the fore plane downlock solution to be reached, at least one of the links in the fore plane is required to deflect. The modelling of this flexibility was presented in Section E. The results of this investigation into the behaviour of the flexible model are presented now.

\section{A. Effect of sidestay attachment point displacement on required downlock loads, with flexible lower sidestay $\mathcal{L}_{3}$}

Figure 10 shows downlock results for the DSS MLG model with one flexible link, the lower sidestay link $\mathcal{L}_{3}$, in the fore plane. Each panel shows a surface of steady-state solutions in terms of the locklink force $F_{l l}$, aft sidestay attachment point deflection $\delta_{a t t}$, and locklink angle $\theta_{4}^{f}$. The case shown in Figure $10(\mathrm{a})$ is the lowest stiffness case considered. When there is no sidestay deflection (i.e. $\delta_{a t t}=0$ ), a given locklink force relates directly to a single gear state. Because the landing gear is semi-locked, the movement of the aft locklinks is practically limited between two steady-state asymptotes. The upper branch of solutions corresponds to the unlocked link state: increasing the force on this branch does not produce a significant change in locklink angle. The lower branch of solutions corresponds to the downlocked link state: decreasing the force on this lower branch does not cause the locklink angle to change much. The locus of equilibria which joins these two branches is approximately linear, and as the only solution when the locklink force is zero is on the lower branch, there is not enough resistance in the structure to prevent the locklinks from reaching the downlocked state under gravitational forces.

As the aft sidestay deflection increases, even only by a few millimetres, the equilibrium curve in the

$\left(F_{l l}, \theta_{4}^{f}\right)$-plane develops two separate areas where the equilibrium curve folds back on itself. In these regions, 

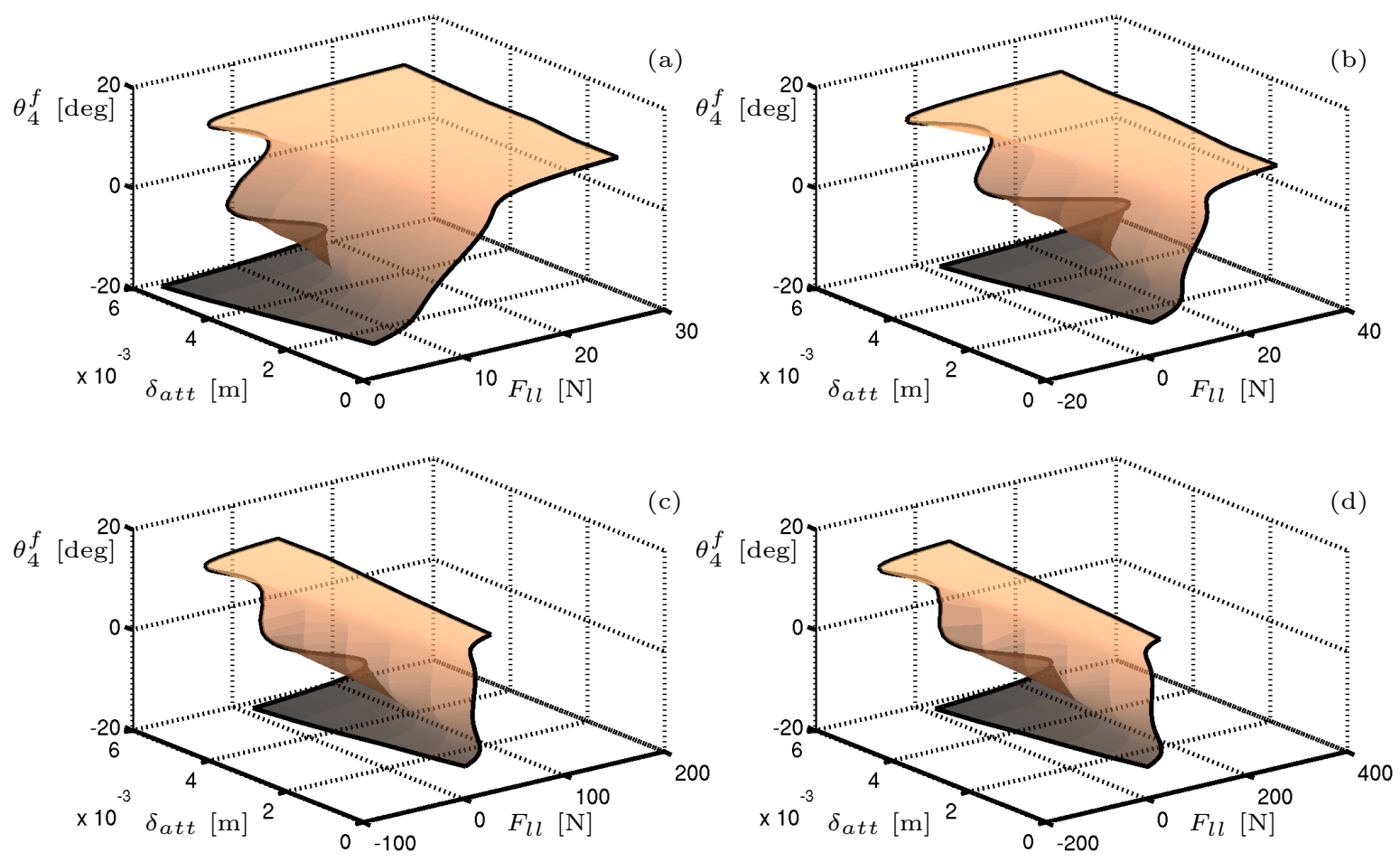

Figure 10: Landing gear response to varying locklink force $F_{l l}$ as a function of aft sidestay attachment point deflection $\delta_{a t t}$, with flexible fore link $\mathcal{L}_{3}$ of stiffness (a) $7.7 \times 10^{4} \mathrm{~N} / \mathrm{m}$, (b) $1.5 \times 10^{5} \mathrm{~N} / \mathrm{m}$, (c) $5.5 \times 10^{5} \mathrm{~N} / \mathrm{m}$, (d) $1.0 \times 10^{6} \mathrm{~N} / \mathrm{m}$. Note the differences in the scale of $F_{l l}$.

a given force corresponds to multiple gear states. For the maximum attachment point deflection considered $\left(\delta_{a t t}=6 \mathrm{~mm}\right)$, the locus of equilibria between the unlocked (upper branch) and downlocked (lower branch) solutions is now highly nonlinear. A double-hysteresis loop has formed as the sidestay attachment point has deflected, such that now there is a range of force values for which there are three or five corresponding gear states. The effect on the DSS MLG's ability to reach the downlocked branch, however, is still fairly minimal, because the upper branch of solutions is only present for positive force values. This sort of behaviour is similar to the results obtained previously ${ }^{12}$ for an overcentre mechanism; however, only a single hysteresis loop is present in the overcentre mechanism response.

Figures 10(b)-(d) show the effect of increased stiffness on the equilibria surfaces. As is shown in Figure 10(b), doubling the stiffness from the initially considered case in Figure 10(a) effectively expands the double-hysteresis loop. Hence, for a given $\delta_{a t t}$ there is a greater range of force values spanning an area with three or five corresponding gear states for a given locklink force. The result of this expansion is that when the attachment point deflection increases beyond about $5.8 \mathrm{~mm}$, the downlock solution cannot be reached from the unlocked position under the action of gravity alone.

By increasing the stiffness further still, the double hysteresis region continues to expand and occur at lower $\delta_{a t t}$ values. For the maximum stiffness considered, shown in Figure 10(d), the downlocked position 
cannot be reached under gravity, even when the sidestay has not deflected at all (i.e. $\delta_{a t t}=0$ ). Even when the attachment point is not deflected, the landing gear is still largely asymmetric ${ }^{\mathrm{b}}$ because the downlocked aft plane is fixed in position. As the lower sidestay's stiffness tends to infinity (i.e. approaches the fully rigid case), the force required to move the locklinks between the unlocked and downlocked states also tends to $\pm \infty$. For a fully rigid DSS MLG model, only two states exist for the landing gear, and the locklinks are no longer able to move between them.

B. Effect of sidestay attachment point displacement on required downlock loads, with flexible upper sidestay $\mathcal{L}_{2}$
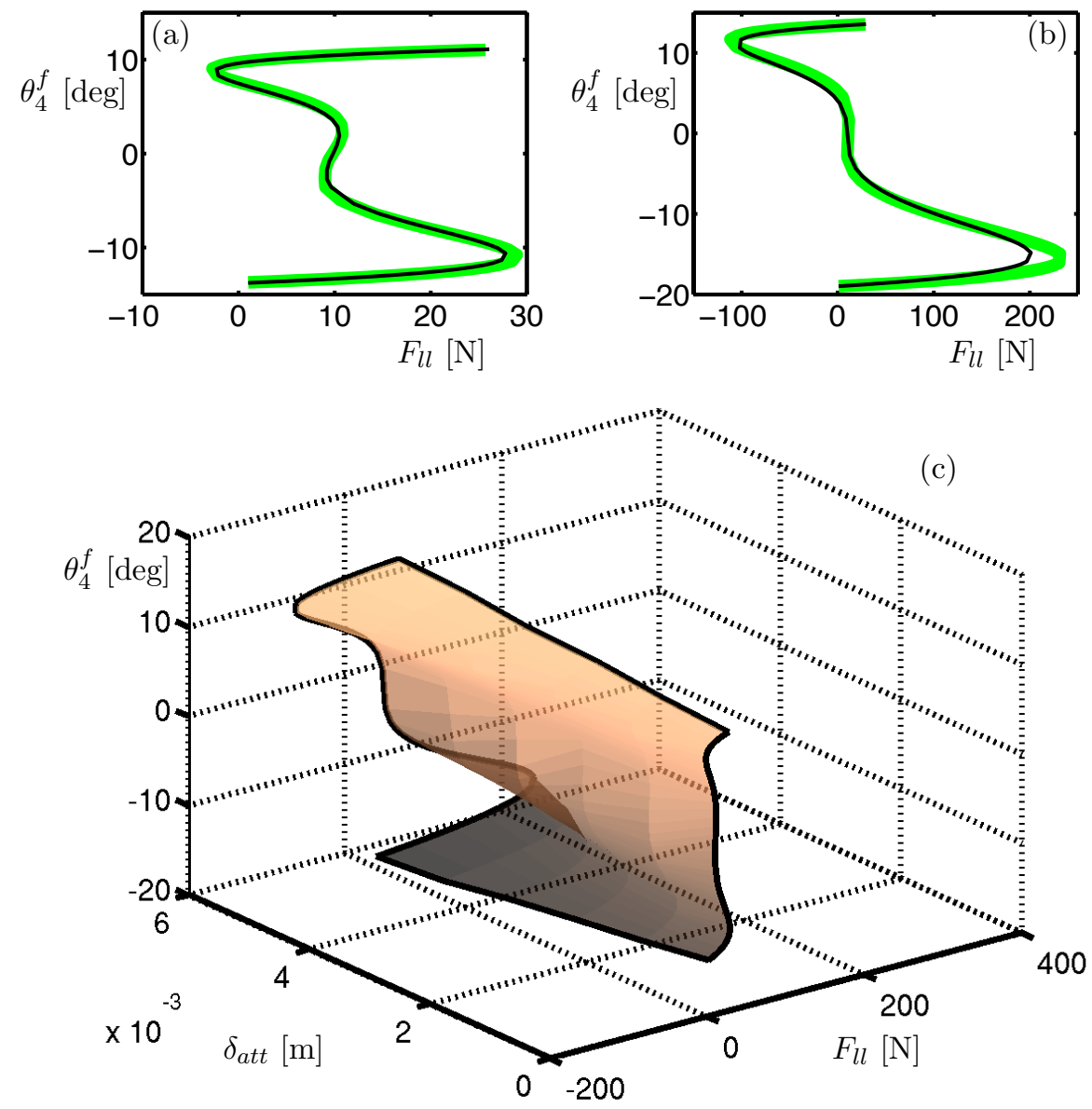

Figure 11: Landing gear with flexible fore link $\mathcal{L}_{2}$ of stiffness $1.0 \times 10^{6} \mathrm{~N} / \mathrm{m}$ response to varying locklink force $F_{l l}$ as a function of fore sidestay attachment point deflections of (a) $\delta_{a t t}=0 \mathrm{~mm}$, (b) $\delta_{a t t}=5.9 \mathrm{~mm}$ and (c) $0 \mathrm{~mm} \leq \delta_{a t t} \leq 5.9 \mathrm{~mm}$. The light curves in (a) and (b) show the equivalent response for the system with a flexible $\mathcal{L}_{3}$.

Figure 11 shows the steady-state surface when the fore upper sidestay $\mathcal{L}_{2}$ is allowed to be flexible whilst all other links remain rigid. The surface in Figure 11(c) is qualitatively similar to the previously considered

\footnotetext{
${ }^{\mathrm{b}}$ There will be two positions where the gear is in a symmetric state but otherwise it is asymmetric
} 
case in Figure 10(d), and upon closer inspection, the quantitative differences between the two are small. The light curves in Figure 11(a) and (b) show equivalent responses to those from Figure 10(d) for comparative purposes. Figure $11(\mathrm{a})$ is the case when $\delta_{a t t}=0$. It shows that when the fore locklinks are unlocked (i.e. on the upper equilibria branch), the response to the locklink force is almost identical to the previous case when $\mathcal{L}_{3}$ is flexible. There is a slight difference around the lower fold point between the two sets of results, indicating that the upper sidestay at this point has to deflect less than the lower sidestay would at the same point.

The difference between the two alternate flexible sidestay cases becomes more pronounced when the attachment point deflection is increased to the case shown in Figure 11(b). The upper and lower fold points on the black curve occur at lower absolute force values than the respective points on the light curve. This provides further evidence that the upper sidestay does not need to deflect as much as the lower sidestay in order to allow the locklinks to move between downlocked and unlocked states. Despite the differences at the upper and lower fold points, the central branch of equilibria between the two internal fold points remains largely unchanged. In fact, there is a small range of equilibria in this region for which attachment point deflection has no noticeable effect. This is due to a combination of two effects. The distance between the two ends of the locklinks hardly changes around the downlock point $\left(\theta_{4}^{f}=0^{\circ}\right)$, so this region is quite insensitive to changes in attachment point position. This insensitivity is coupled with the sidestay force direction of action: the majority of the internal forces in the sidestays act perpendicularly to the locklinks when they are approximately aligned around the downlock point. These two effects result in the very small linear region around $\theta_{4}^{f}=0^{\circ}$ which remains unchanged with increasing attachment point deflections. It should be noted that the apparent change in this region between Figures $11(\mathrm{a})$ and (b) are a result of the changing $F_{l l}$-scale.

One advantage of using the numerical continuation approach over dynamic simulations is highlighted by considering this double-hysteresis behaviour. A dynamic simulation, starting from an unlocked solution, with a slowly decreasing locklink force would approximately trace out the upper branch of equilibria, jumping to the lower branch when the system reaches the upper fold point. From the lower branch, slowly increasing the downlock force will approximately trace out the lower branch of equilibria until reaching the lower fold point, where it will jump to the upper branch. It would be very difficult to identify the central equilibria with dynamic simulations, and so it is likely that this region would be missed.

However, the reason for this jump is a change in the dynamic stability of the underlying equilibria. Formulating the mechanism equations as coupled, steady-state equations is necessary to be able to apply numerical continuation techniques, however these equations do not contain any information on the dynamic stability of solutions. This stability information has to be inferred through the appropriate use of dynamic simulations, coupled with some knowledge 

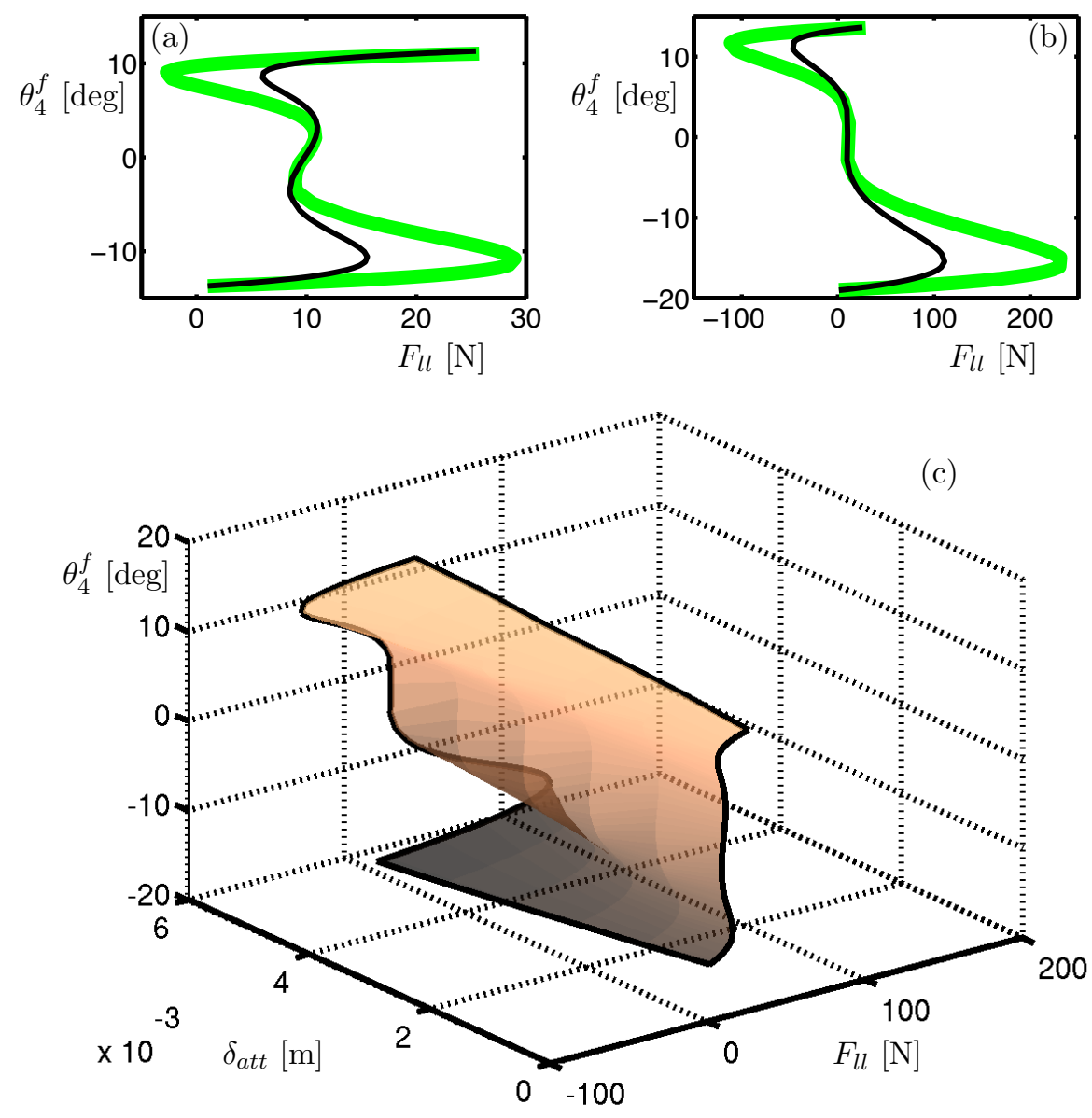

Figure 12: Landing gear with flexible fore links $\mathcal{L}_{2}$ and $\mathcal{L}_{3}$ of stiffnesses $k_{2}=k_{3}=1.0 \times 10^{6} \mathrm{~N} / \mathrm{m}$ response to varying locklink force $F_{l l}$ as a function of aft sidestay attachment point deflections of (a) $\delta_{a t t}=0 \mathrm{~mm}$, (b) $\delta_{a t t}=5.9 \mathrm{~mm}$ and (c) $0 \mathrm{~mm} \leq \delta_{a t t} \leq 5.9 \mathrm{~mm}$. The light curves in (a) and (b) show the equivalent response for the system with a flexible $\mathcal{L}_{3}$.

of the system behaviour. Previous work studying an overcentre mechanism ${ }^{12}$ revealed hysteresis behaviour observed when a resistive force is applied between a two-link mechanism (similar to considering the locklinks in isolation). In light of this prior work, it is reasoned that the uppermost and lowermost branches are dynamically stable. This means that nearby solutions will tend towards these two branches over time, so the equilibria can be traced dynamically in parameter space provided the parameter variation is sufficiently slow (quasi-static). This quasi-static assumption is reasonable for a landing gear locking mechanism. 


\section{Effect of sidestay attachment point displacement on required downlock loads, with flexible upper and lower sidestays $\mathcal{L}_{2}$ and $\mathcal{L}_{3}$}

Figure 12 compares the result when both $\mathcal{L}_{2}$ and $\mathcal{L}_{3}$ are flexible, with the baseline case of $\mathcal{L}_{3}$ as the only flexible link. The forces at the upper and lower fold points on the black curves are much lower in magnitude than the baseline, light curve case. The reason for this significant difference is that the sidestay-locklink joint, point $C$, is no longer confined to lie on an arc defined by the rigid link. For the baseline case shown by the light curves, point $C$ was still constrained to lie a fixed distance from the fore attachment point $A$. The case with a flexible $\mathcal{L}_{2}$ in Figure 11 is similar to the baseline case, as the sidestay-locklink joint at point $C$ is still constrained to lie a fixed distance from the lower sidestay joint, point $B$. This results in similar magnitudes of locklink force at the fold points for both of the single stiffness cases. With both upper and lower fore sidestays allowed to flex axially, the sidestay-locklink joint at point $C$ is not constrained to lie on any one path. Hence, the system can take the lowest energy position, which will result in point $C$ tracing out a path somewhere between the two arcs made by $\mathcal{L}_{2}$ about the attachment point $A$ and $\mathcal{L}_{3}$ about the lower sidestay joint $B$.
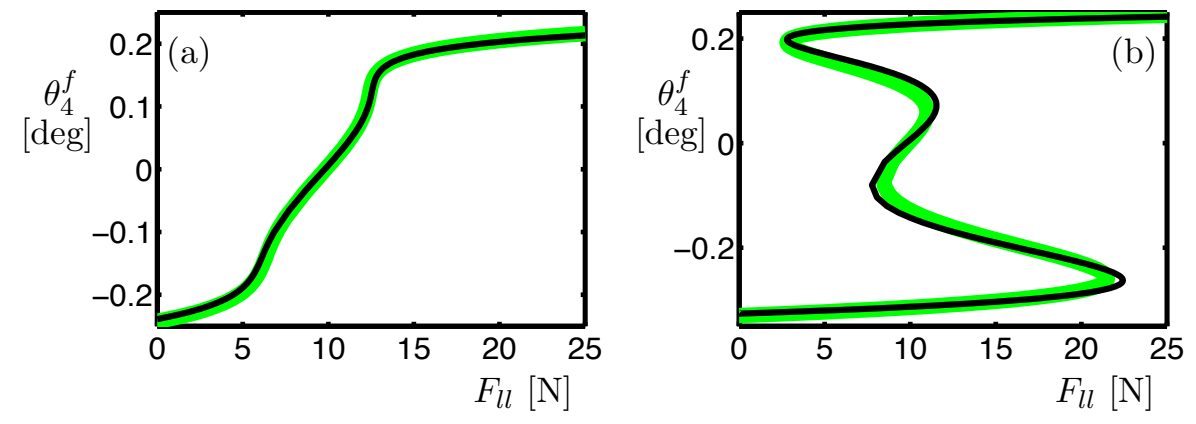

Figure 13: Landing gear response to fore sidestay attachment point deflections of (a) $\delta_{a t t}=0 \mathrm{~mm}$ and (b) $\delta_{a t t}=5.9 \mathrm{~mm}$. The light curves show the response when $k_{2}=2.0 \times 10^{6} \mathrm{~N} / \mathrm{m}$ and $k_{3}=1.0 \times 10^{5} \mathrm{~N} / \mathrm{m}$, and the black curves show the response when $k_{2}=1.0 \times 10^{5} \mathrm{~N} / \mathrm{m}$ and $k_{3}=2.0 \times 10^{6} \mathrm{~N} / \mathrm{m}$.

Figure 13 compares the responses of the landing gear when the upper and lower sidestays have different stiffnesses. The black curves are for the case when the lower sidestay $\mathcal{L}_{3}$ has a higher stiffness than the upper sidestay $\mathcal{L}_{2}$, and the light curves are for the opposite case when the upper sidestay stiffness is greater than the lower sidestay stiffness. In Figure 13(a) there is very little difference between the two curves. The case shown by the light curve (with a high $\mathcal{L}_{2}$ stiffness) requires slightly more force to hold the gear at the same geometric configuration (i.e. at the same locklink angle). This difference becomes more pronounced when the attachment point is deflected to about $6 \mathrm{~mm}$, shown in Figure 13(b). This suggests that the upper sidestay needs to extend less than the lower sidestay for the locklinks to move between unlocked and downlocked positions, because of the significance of relative stiffnesses. For the light curve, the upper sidestay is stiffer 
than the lower sidestay, so the lower sidestay will deflect more when the locklinks move between the unlocked and downlocked branches. Moving between the unlocked and downlocked states requires the most force for this case. For the opposite scenario (i.e. the lower sidestay is stiffer), shown by the black curve in Figure 13, the upper sidestay will deflect more than the lower sidestay as the locklinks move through the downlock point. Because this case of predominantly upper-sidestay deflection results in a lower force at the upper and lower fold points than the predominantly lower-sidestay deflection case, it suggests that the lower force is a result of a smaller deflection of the upper sidestay. This result could be used to tailor the sidestay stiffnesses such that the upper sidestay provides less structural resistance (i.e. has a lower stiffness) than the lower sidestay, to reduce the energy barrier to the downlocked position.

\section{Concluding Remarks}

It has been shown how a three-dimensional dual-sidestay main landing gear mechanism can be modelled as a set of fully parameterised steady-state constraint equations. The relatively simple method behind the equation formulation provides a flexible approach to analysing complex mechanisms, whilst being well-suited for the use of numerical methods from bifurcation theory. Furthermore, the equations can be adjusted in a straightforward manner to model structural flexibilities within the sidestays of the landing gear mechanism.

The suitability of this modelling and analysis approach was demonstrated with an investigation into the effects of sidestay angle upon retraction actuator loads. The geometry of the landing gear can be changed simply by numerically continuing an assigned sidestay angle parameter. This proved highly advantageous because it avoids a need to re-formulate the model to cope with the different geometries considered as the sidestay was continued from the single sidestay case to the symmetric dual sidestay case. The results agreed very well with a previous model of a single sidestay three-dimensional MLG, which constitutes a validation of the DSS MLG model. The sidestay angle was found to play an important role in determining the extent to which the landing gear can retract. The geometry of the sidestay and locklinks was found to prevent the landing gear from retracting fully, especially for the case where the sidestay being moved was in the planar position (i.e. the sidestay plane angle was zero). The retraction actuator loads, however, were found to be largely unaffected by sidestay angle.

An investigation was then conducted into the sensitivity of the locklinks to sidestay attachment point deflections. It was discovered that the underlying steady-state behaviour developed a double-hysteresis loop as the sidestay attachment point was deflected, and that the downlock force is highly sensitive to attachment point deflections of only a few millimetres. This was exacerbated by increasing the stiffnesses of the sidestays.

For the geometry considered, it appears that a relatively low upper sidestay stiffness (when compared to the lower sidestay stiffness) would be beneficial in enabling the locklinks to move between the unlocked and 
downlocked states.

Future modelling work on dual-sidestay landing gear mechanisms could include adding more flexible elements within the model. Around the downlock point, the sidestay deflections would also cause the shock strut to deflect. This deflection would depend on the relative forces entering the strut from both the fore and aft sidestays. Another aspect not captured by the current model, which could be added for future investigations, is a specific rotation axis for the upper fore and aft sidestays to rotate about. In the current model, the sidestay plane is defined in part by the vector from the origin to the sidestay attachment point, so when the attachment point is deflected this vector automatically rotates. In a real landing gear, this axis is initially set so that it runs from the nominal sidestay attachment point to the co-ordinate origin, but that does not necessarily hold true when the sidestay attachment point deflects. This difference is significant because, if the sidestay rotation axis does not intersect the origin, the mechanism will become stuck, requiring structural deflections to allow motion to continue.

\section{References}

${ }^{1}$ SAFRAN group, Messier-Dowty Exhibits Wide Range of Landing Gear Technology at Paris Air Show, , accessed on $18 / 01 / 2012$

${ }^{2}$ FlightGlobal.com, Airbus adopts Vickers VC10 landing gear concept for A350 XWB, http://www.flightglobal.com/news/articles/pictures-airbus-adopts-vickers-vc10-landing-gear-concept-for-a350-xwb-214238/ , accessed on $18 / 01 / 2012$

${ }^{3}$ Conway, H.G., Landing Gear Design. Chapman and Hall, London, 1958.

${ }^{4}$ Currey, N.S., Aircraft Landing Gear Design: Principles and Practices. AIAA, Washington D.C, 1988.

${ }^{5}$ Lyle, K.H., Jackson, K.E., Fasanella, E.L., Simulation of Aircraft Landing Gears with a Nonlinear Dynamic Finite Element Code, AIAA Journal of Aircraft, Vol. 39, No. 1, January - February 2002.

${ }^{6}$ Kruger, W., Besselink, I., Cowling, D., Doan, D.B., Kortum, W., Krabacher, W., Aircraft Landing Gear Dynamics: Simulation and Control, Vehicle System Dynamics, Vol. 28, 1997.

${ }^{7}$ Daniels, J. N., A Method for Landing Gear Modeling and Simulation With Experimental Validation NASA Contractor Report 201601, 1996

${ }^{8}$ Ghiringhelli, G. L., Gualdi, S., Boschetto, M., Bianco-Mengotti, R., Analysis of Landing Gear Behaviour for Trainer Aircraft $15^{\text {th }}$ European ADAMS Users' Conference, Rome, 2000

${ }^{9}$ Strogatz, S., Nonlinear dynamics and chaos, Springer, 2000.

${ }^{10}$ Guckenheimer, J. and Holmes, P., Nonlinear Oscillations, Dynamical Systems and Bifurcations of Vector Fields, Applied Mathematical Sciences Vol. 42, Westview Press, February 2002.

${ }^{11}$ Krauskopf, B., Osinga, H. M., and Galàn-Vioque, J., Numerical Continuation Methods for Dynamical Systems, Springer, 2007.

${ }^{12}$ Knowles, J.A.C., Krauskopf, B., Lowenberg, M.H., Numerical Continuation Applied to Landing Gear Mechanism Analysis, AIAA Journal of Aircraft, Vol. 49, No. 8, July - August 2011. 
${ }^{13}$ Rankin, J., Coetzee, E., Krauskopf, B. and Lowenberg, M. H., Bifurcation and Stability Analysis of Aircraft Turning on the Ground, AIAA Journal of Guidance, Dynamics and Control, Vol. 32, No. 2, March 2009.

${ }^{14}$ Thota, P., Krauskopf, B. and Lowenberg, M. H., Interaction of Torsion and Lateral Bending in Aircraft Nose Landing Gear Shimmy, Nonlinear Dynamics, 57(3), 2009.

${ }^{15}$ Knowles, J. A. C., Krauskopf, B. and Lowenberg, M. H., Numerical Continuation of a Three-dimensional Main Landing Gear Mechanism, Submitted to Nonlinear Dynamics [Preprint], May 2012

${ }^{16}$ Doedel, E., Champneys, A., Fairgrieve, T., Kuznetsov, Y., Sandstede, B., and Wang, X., AUTO 97 : Continuation and bifurcation software for ordinary differential equations, http://indy.cs.concordia.ca/auto/, May 2001.

${ }^{17}$ Hoerner, S. F., Aerodynamic Drag, Ohio, 1951 


\section{Appendix I - Model Matrices}

Here, the matrices used in Equation (27) are given. Angles $\Omega_{1-4}$ are depicted in Figure 3, and all other notation is as previously defined. The fore and aft force coefficient matrices $A^{f}$ and $A^{a}$ are $(18 \times 19)$ and $(18 \times 18)$ matrices, respectively. They are multiplied by the force vectors $F^{f}$ and $F^{a}$, which are $(1 \times 19)$ and $(1 \times 18)$ vectors, respectively. The locklink force $F_{\text {unlock }}$ is applied in the fore plane, which is why the fore plane force vector is one element longer than the aft plane force vector. The fore and aft force coefficient matrices and force vectors are (where $s \equiv$ sin, $c \equiv$ cos): 


$$
\begin{aligned}
& A^{f}=\left[\begin{array}{cccccccccccccccccccc}
-L_{2} s \theta_{2}^{f} & L_{2} c \theta_{2}^{f} & 0 & 0 & 0 & 0 & 0 & 0 & 0 & 0 & 0 & 0 & 0 & 0 & 0 & 0 & 0 & 0 & 0 \\
0 & 0 & 1 & 0 & 1 & 0 & 0 & 0 & 0 & 0 & 0 & 0 & 0 & 0 & 0 & 0 & 0 & 0 & 0 \\
0 & 0 & 0 & 1 & 0 & 1 & 0 & 0 & 0 & 0 & 0 & 0 & 0 & 0 & 0 & 0 & 0 & 0 & 0 \\
0 & 0 & 0 & 0 & -L_{3} s \theta_{3}^{f} & L_{3} c \theta_{3}^{f} & 0 & 0 & 0 & 0 & 0 & 0 & 0 & 0 & 0 & 0 & 0 & 0 & 0 \\
0 & 0 & 0 & 0 & 0 & 0 & 1 & 0 & 1 & 0 & 0 & 0 & 0 & 0 & 0 & 0 & 0 & 0 & 0 \\
0 & 0 & 0 & 0 & 0 & 0 & 0 & 1 & 0 & 1 & -1 & 0 & 0 & 0 & 0 & 0 & 0 & 0 & 0 \\
0 & 0 & 0 & 0 & 0 & 0 & 0 & 0 & L_{4} s \theta_{4}^{f} & -L_{4} c \theta_{4}^{f} & L_{4} c \theta_{4}^{f} & 0 & 0 & 0 & 0 & 0 & 0 & 0 & 0 \\
0 & 0 & 0 & 0 & 0 & 0 & 0 & 0 & 0 & 0 & 0 & 1 & 0 & 1 & 0 & 0 & 0 & 0 & 0 \\
0 & 0 & 0 & 0 & 0 & 0 & 0 & 0 & 0 & 0 & 0 & 0 & 1 & 0 & 1 & 0 & 0 & 0 & 0 \\
1 & 0 & 1 & 0 & 0 & 0 & 1 & 0 & 0 & 0 & 0 & 0 & 0 & 0 & 0 & 0 & 0 & 0 & 0 \\
0 & 1 & 0 & 1 & 0 & 0 & 0 & 1 & 0 & 0 & 0 & 0 & 0 & 0 & 0 & 0 & 0 & 0 & 0 \\
0 & 0 & 0 & 0 & 0 & 0 & 0 & 0 & 1 & 0 & 0 & 1 & 0 & 0 & 0 & 0 & 0 & 0 & 0 \\
0 & 0 & 0 & 0 & 0 & 0 & 0 & 0 & 0 & 1 & 0 & 0 & 1 & 0 & 0 & 0 & 0 & 0 & 0 \\
0 & 0 & 0 & 0 & 0 & 0 & 0 & 0 & 0 & 0 & 0 & 0 & 0 & t_{2,2}^{f} & t_{2,3}^{f} & 0 & 0 & -1 & 0 \\
0 & 0 & 0 & 0 & 0 & 0 & 0 & 0 & 0 & 0 & 0 & 0 & 0 & t_{3,2}^{f} & t_{3,3}^{f} & 0 & 0 & 0 & -1 \\
0 & 0 & 0 & 0 & t_{2,2}^{f} & t_{2,3}^{f} & 0 & 0 & 0 & 0 & 0 & 0 & 0 & 0 & 0 & -1 & 0 & 0 & 0 \\
0 & 0 & 0 & t_{3,2}^{f} & t_{3,3}^{f} & 0 & 0 & 0 & 0 & 0 & 0 & 0 & 0 & 0 & 0 & -1 & 0 & 0
\end{array}\right], \\
& \bar{F}^{f}=-\left[F_{2 ; 3,4}^{y} F_{2 ; 3,4}^{z} F_{3 ; 2,4}^{y} F_{3 ; 2,4}^{z} F_{3 ; 1}^{y} F_{3 ; 1}^{z} F_{4 ; 2,3}^{y} F_{4 ; 2,3}^{z} F_{4 ; 5}^{y} F_{4 ; 5}^{z} F_{l l} F_{5 ; 4}^{y} F_{5 ; 4}^{z} F_{5 ; 1}^{y} F_{5 ; 1}^{z} \mathcal{F}_{1 ; 3}^{y} \mathcal{F}_{1 ; 3}^{z} \mathcal{F}_{1 ; 5}^{y} \mathcal{F}_{1 ; 5}^{z}\right]^{T},
\end{aligned}
$$




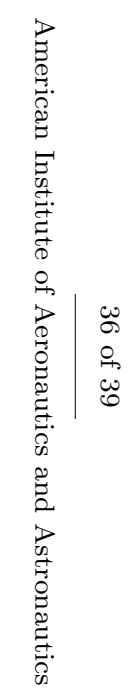

$$
\begin{aligned}
& A^{a}=\left[\begin{array}{cccccccccccccccccc}
-L_{6} s \theta_{6}^{a} & L_{6} c \theta_{6}^{a} & 0 & 0 & 0 & 0 & 0 & 0 & 0 & 0 & 0 & 0 & 0 & 0 & 0 & 0 & 0 & 0 \\
0 & 0 & 1 & 0 & 1 & 0 & 0 & 0 & 0 & 0 & 0 & 0 & 0 & 0 & 0 & 0 & 0 & 0 \\
0 & 0 & 0 & 1 & 0 & 1 & 0 & 0 & 0 & 0 & 0 & 0 & 0 & 0 & 0 & 0 & 0 & 0 \\
0 & 0 & 0 & 0 & -L_{7} s \theta_{7}^{a} & L_{7} c \theta_{7}^{a} & 0 & 0 & 0 & 0 & 0 & 0 & 0 & 0 & 0 & 0 & 0 & 0 \\
0 & 0 & 0 & 0 & 0 & 0 & 1 & 0 & 1 & 0 & 0 & 0 & 0 & 0 & 0 & 0 & 0 & 0 \\
0 & 0 & 0 & 0 & 0 & 0 & 0 & 1 & 0 & 1 & 0 & 0 & 0 & 0 & 0 & 0 & 0 & 0 \\
0 & 0 & 0 & 0 & 0 & 0 & 0 & 0 & L_{8} s \theta_{8}^{a} & -L_{8} c \theta_{8}^{a} & 0 & 0 & 0 & 0 & 0 & 0 & 0 & 0 \\
0 & 0 & 0 & 0 & 0 & 0 & 0 & 0 & 0 & 0 & 1 & 0 & 1 & 0 & 0 & 0 & 0 & 0 \\
0 & 0 & 0 & 0 & 0 & 0 & 0 & 0 & 0 & 0 & 0 & 1 & 0 & 1 & 0 & 0 & 0 & 0 \\
1 & 0 & 0 & 0 & 0 & 0 & 0 & 0 & 0 & 0 & 0 & 0 & -L_{9} s \theta_{9}^{a} & L_{9} c \theta_{9}^{a} & 0 & 0 & 0 & 0 \\
0 & 1 & 0 & 1 & 0 & 0 & 0 & 1 & 0 & 0 & 0 & 0 & 0 & 0 & 0 & 0 & 0 & 0 \\
0 & 0 & 0 & 0 & 0 & 0 & 0 & 0 & 1 & 0 & 1 & 0 & 0 & 0 & 0 & 0 & 0 & 0 \\
0 & 0 & 0 & 0 & 0 & 0 & 0 & 0 & 0 & 1 & 0 & 1 & 0 & 0 & 0 & 0 & 0 & 0 \\
0 & 0 & 0 & 0 & 0 & 0 & 0 & 0 & 0 & 0 & 0 & 0 & t_{2,2}^{a} & t_{2,3}^{a} & 0 & 0 & -1 & 0 \\
0 & 0 & 0 & 0 & 0 & 0 & 0 & 0 & 0 & 0 & 0 & 0 & t_{3,2}^{a} & t_{3,3}^{a} & 0 & 0 & 0 & -1 \\
0 & 0 & 0 & 0 & t_{2,2}^{a} & t_{2,3}^{a} & 0 & 0 & 0 & 0 & 0 & 0 & 0 & 0 & -1 & 0 & 0 & 0 \\
0 & 0 & t_{3,2}^{a} & t_{3,3}^{a} & 0 & 0 & 0 & 0 & 0 & 0 & 0 & 0 & 0 & -1 & 0 & 0
\end{array}\right], \\
& \bar{F}^{a}=-\left[F_{6 ; 7,8}^{y} F_{6 ; 7,8}^{z} F_{7 ; 6,8}^{y} F_{7 ; 6,8}^{z} F_{7 ; 1}^{y} F_{7 ; 1}^{z} F_{8 ; 6,7}^{y} F_{8 ; 6,7}^{z} F_{8 ; 9}^{y} F_{8 ; 9}^{z} F_{9 ; 8}^{y} F_{9 ; 8}^{z} F_{9 ; 1}^{y} F_{9 ; 1}^{z} \mathcal{F}_{1 ; 7}^{y} \mathcal{F}_{1 ; 7}^{z} \mathcal{F}_{1 ; 9}^{y} \mathcal{F}_{1 ; 9}^{z}\right]^{T},
\end{aligned}
$$


The non-force-coefficient terms are, in the fore plane:

$$
\bar{B}^{f}=-\left[\begin{array}{l}
\frac{L_{2}}{2} m_{2} g_{z} \cos \theta_{2}^{f}-\frac{L_{2}}{2} m_{2} g_{y} \sin \theta_{2}^{f} \\
m_{3} g_{y} \\
m_{3} g_{z} \\
\frac{L_{3}}{2} m_{3} g_{z} \cos \theta_{3}^{f}-\frac{L_{3}}{2} m_{3} g_{y} \sin \theta_{3}^{f} \\
m_{4} g_{y} \\
m_{4} g_{z} \\
-\frac{L_{4}}{2} m_{4} g_{z} \cos \theta_{4}^{f}+\frac{L_{4}}{2} m_{4} g_{y} \sin \theta_{4}^{f} \\
m_{5} g_{y} \\
m_{5} g_{z} \\
\frac{L_{5}}{2} m_{5} g_{z} \cos \theta_{5}^{f}-\frac{L_{5}}{2} m_{5} g_{y} \sin \theta_{5}^{f} \\
0 \\
0 \\
0 \\
-t_{2,1}^{f} F_{5 ; 1}^{f} F_{3 ; 1}^{x} \\
0 \\
0 \\
0
\end{array}\right],
$$


in the aft plane:

$$
\bar{B}^{a}=-\left[\begin{array}{l}
\frac{L_{6}}{2} m_{6} g_{z} \cos \theta_{6}^{a}-\frac{L_{6}}{2} m_{6} g_{y} \sin \theta_{6}^{a} \\
m_{7} g_{y} \\
m_{7} g_{z} \\
\frac{L_{7}}{2} m_{7} g_{z} \cos \theta_{7}^{a}-\frac{L_{7}}{2} m_{7} g_{y} \sin \theta_{7}^{a} \\
m_{8} g_{y} \\
m_{8} g_{z} \\
-\frac{L_{8}}{2} m_{8} g_{z} \cos \theta_{8}^{a}+\frac{L_{8}}{2} m_{8} g_{y} \sin \theta_{8}^{a} \\
m_{9} g_{y} \\
m_{9} g_{z} \\
\frac{L_{9}}{2} m_{9} g_{z} \cos \theta_{9}^{a}-\frac{L_{9}}{2} m_{9} g_{y} \sin \theta_{9}^{a} \\
0 \\
0 \\
-t_{3,1}^{a} F_{7 ; 1}^{x} \\
0 \\
-t_{2,1}^{a} F_{9 ; 1}^{x} F_{9 ; 1}^{x} \\
0 \\
\end{array}\right],
$$

and in the global $(Y, Z)$-plane:

$$
\begin{aligned}
B^{\mathcal{L}_{1}}= & \left(\frac{m_{1}}{2} G_{z}+m_{\text {wheel }}\right) L_{1} \cos \Theta_{1}+\mathcal{F}_{\text {act }}^{z}\left(l_{1 a c t} \cos \left(\Theta_{1}+\Omega_{5}\right)\right) \\
& -\mathcal{F}_{\text {act }}^{y}\left(l_{1 a c t} \sin \left(\Theta_{1}+\Omega_{5}\right)\right)+M_{D}-M
\end{aligned}
$$


The row vector $C$ is given as

$$
\left[\begin{array}{c}
C_{1} \\
\vdots \\
C_{16} \\
C_{17} \\
C_{18} \\
C_{19} \\
C_{20} \\
\vdots \\
C_{34} \\
C_{35} \\
C_{36} \\
C_{37}
\end{array}\right]^{T}=\left[\begin{array}{c}
0 \\
\vdots \\
\frac{L_{1}}{2} \sin \Theta_{1}-l_{13} \sin \left(\Theta_{1}+\Omega_{1}\right) \\
l_{13} \cos \left(\Theta_{1}+\Omega_{1}\right)-\frac{L_{1}}{2} \cos \Theta_{1} \\
\frac{L_{1}}{2} \sin \Theta_{1}-l_{15} \sin \left(\Theta_{1}-\Omega_{2}\right) \\
l_{15} \cos \left(\Theta_{1}-\Omega_{2}\right)-\frac{L_{1}}{2} \cos \Theta_{1} \\
\vdots \\
\frac{L_{1}}{2} \sin \Theta_{1}-l_{17} \sin \left(\Theta_{1}+\Omega_{3}\right) \\
l_{17} \cos \left(\Theta_{1}+\Omega_{3}\right)-\frac{L_{1}}{2} \cos \Theta_{1} \\
\frac{L_{1}}{2} \sin \Theta_{1}-l_{19} \sin \left(\Theta_{1}-\Omega_{4}\right) \\
l_{19} \cos \left(\Theta_{1}-\Omega_{4}\right)-\frac{L_{1}}{2} \cos \Theta_{1}
\end{array}\right]
$$

Here $M$ is the retraction actuator moment parameter, $\mathcal{F}_{\text {act }}$ is the actuator force parameter (both initially chosen to be zero to reflect the deployed MLG state) and $M_{D}$ is the drag-induced moment (set to zero in this work); length $l_{1 a c t}$ is the distance from the shock strut cg to the adjoining retraction actuator, and $\Omega_{5}$ is the angle that the length $l_{1 a c t}$ makes with the shock strut centreline (in local co-ordinates). 